Type inference for unique pattern matching

Peer-reviewed author version

VANSUMMEREN, Stijn (2006) Type inference for unique pattern matching. In: ACM

TRANSACTIONS ON PROGRAMMING LANGUAGES AND SYSTEMS, 28(3). p. 389-428.

Handle: http://hdl.handle.net/1942/1425 


\title{
Type Inference for Unique Pattern Matching
}

\author{
STIJN VANSUMMEREN \\ Limburgs Universitair Centrum
}

\begin{abstract}
Regular expression patterns provide a natural, declarative way to express constraints on semistructured data and to extract relevant information from it. Indeed, it is a core feature of the programming language Perl, surfaces in various UNIX tools such as sed and awk, and has recently been proposed in the context of the XML programming language XDuce. Since regular expressions can be ambiguous in general, different disambiguation policies have been proposed to get a unique matching strategy. We formally define the matching semantics under both (1) the POSIX, and (2) the first and longest match disambiguation strategies. We show that the generally accepted method of defining the longest match in terms of the first match and recursion does not conform to the natural notion of longest match. We continue by solving the type inference problem for both disambiguation strategies, which consists of calculating the set of all subparts of input values a subexpression can match under the given policy.
\end{abstract}

Categories and Subject Descriptors: D.3.3 [Programming Languages]: Language Constructs and Features-patterns; F.3.2 [Logics and Meanings of Programs]: Semantics of Programming Languages-program analysis; F.4.3 [Mathematical Logic and Formal Languages]: Formal Languages - classes defined by grammars or automata (e.g., context-free languages, regular sets, recursive sets); operations on languages; H.2.3 [Database Management]: Languagesquery languages; XML

General Terms: Design, languages, theory, verification

Additional Key Words and Phrases: pattern matching, disambiguation policies, programming languages, XML

\section{INTRODUCTION}

The Extensible Markup Language (XML) [Yergeau et al. 2004] provides a standard syntax for describing tree-structured and semi-structured data. In the past few years it has become the standard format for the representation and exchange of data on the web. Although XML can describe arbitrary trees, most applications restrict themselves to a set of valid trees, described by a schema. The standard schema language promoted by the World Wide Web Consortium (W3C) is XML Schema [Thompson et al. 2001], although various other schema languages exist [Davidson et al. 1999; Clark and Makoto 2001; Møller 2003].

Recently, there has been growing interest to make XML transformations type safe: given a schema for the input trees, does the transformed output tree always adhere to some output schema [Suciu 2002]? One of the most influential treatments

The author is a Research Assistant of the Fund for Scientific Research - Flanders.

Author's address: Stijn Vansummeren, Limburgs Universitair Centrum, Universitaire Campus, Gebouw D, B-3590 Diepenbeek, Belgium.

Permission to make digital/hard copy of all or part of this material without fee for personal or classroom use provided that the copies are not made or distributed for profit or commercial advantage, the ACM copyright/server notice, the title of the publication, and its date appear, and notice is given that copying is by permission of the ACM, Inc. To copy otherwise, to republish, to post on servers, or to redistribute to lists requires prior specific permission and/or a fee. 
of this typechecking problem was done by Hosoya et al., in the context of the XML programming language XDuce [Hosoya 2000; Hosoya and Pierce 2003; Hosoya et al. 2005]. They introduced a type system of regular expression types based on regular tree languages capable of expressing XML Schema, and gave an efficient subtyping algorithm. XDuce's type system has strongly influenced that of XQuery [Boag et al. 2005], the standard XML query language of the W3C.

In addition, XDuce proposed an extension of ML-style patterns, called regular (hedge) expression patterns to support data extraction on hedges (sequences of trees) [Hosoya 2000; Hosoya and Pierce 2002]. In order to support such patterns in a statically typed programming language, Hosoya and Pierce argued that the compiler has to infer the types of the variable bindings occurring in a pattern, otherwise the type annotations become too heavy.

The idea of regular expression pattern matching stems from traditional string manipulation languages such as Perl, and UNIX tools such as sed and awk [Dougherty and Robbins 1996]. These languages remain in frequent use today, as a lot of legacy semi-structured data is not tree-structured, but consists of ordinary string content. None of the above languages can guarantee the type safety of a transformation however. A study of regular expression pattern matching for strings and its associated type inference problem is hence an important first step towards type safe string transformations in those languages.

Syntactically, regular (hedge) expression patterns are regular (hedge) expressions annotated with variable binders. In general, regular expressions can be ambiguous, meaning that there are various ways of matching the input, resulting in multiple possible bindings of the variables. In order to obtain a unique matching semantics, one therefore needs to disallow ambiguous patterns [Book et al. 1971; Hosoya 2003], or define a disambiguation policy. Various disambiguation policies exist, and it is currently unclear which one is to be preferred:

- the XDuce policy, also employed by Perl;

- the first and longest match; and

- the POSIX policy, employed by all IEEE POSIX compliant tools, including sed and awk.

Especially the XDuce policy and its related type inference problem has been extensively studied. It was introduced by Hosoya and Pierce [Hosoya 2000; Hosoya and Pierce 2002], who also developed its first type inference algorithm. This algorithm is imprecise however, since it only computes precise types for tail variables. A precise algorithm was later developed in the context of the XML-centric general-purpose programming language $\mathbb{C D u c e}$ [Frisch et al. 2002; 2003]. Both approaches consider the policy in a hedge-based setting. A type inference algorithm for the string-based setting was developed at the same time by Tabuchi et al. [2002].

The first and longest match policy was also (indirectly) introduced by Hosoya and Pierce [2000; 2002], as a means to intuitively explain the XDuce policy. We will show, however, that this generally accepted intuition is false. As a consequence, the first and longest match policy has not been studied before. To our knowledge, the POSIX policy [Institute of Electrical and Electronic Engineers 1992] has not been studied before either. 
In this paper we will formalize the POSIX and first and longest match disambiguation policies for both strings and hedges, and develop precise type inference algorithms for them. Our aim here is to treat strings and hedges in a uniform manner, and to develop declarative type inference algorithms which specify the formal languages that need to be calculated, but do not rely on a concrete implementation strategy. This approach has two benefits: we get a better understanding of the the fundamental difficulties involved, and our solutions can be integrated in existing regular language frameworks (such as that of MONA [Elgaard et al. 1998; Klarlund and Møller 2001], XDuce, or $\mathbb{C D u c e}$ ).

We note that we are the first to demonstrate soundness and completeness of a type inference algorithm for regular expression pattern matching. Indeed, the XDuce and $\lambda^{\text {re }}$ algorithms are imprecise [Hosoya and Pierce 2002; Hosoya 2000; Sumii 2003], while the correctness proof of $\mathbb{C D u c e}$ is unpublished.

The rest of this paper is organized as follows. Section 2 introduces regular expression pattern matching, the importance of a disambiguation strategy to get a unique match, and the type inference problem. We formally define regular expression string patterns in Section 3. We then define the matching relation on strings according to the POSIX policy in Section 4, and solve its associated type inference problem in Section 5. The insights gained will help us formalize the matching relation on strings according to the first and longest match policy in Section 6 where we also discuss its difference with the XDuce policy. We then develop a precise type inference algorithm in Section 7. Section 8 introduces regular hedge expression patterns. Finally, we show how the matching process under the first and longest match policy and its associated type inference problem can be lifted to the hedge-based setting in Sections 9 and 10. The last section provides discussion and some pointers to future work.

\section{BASIC CONCEPTS}

\subsection{Pattern Matching}

Pattern matching in declarative programming languages such as Prolog [Sterling and Shapiro 1994] or ML [Ullman 1998] provides a means to describe constraints on values, at the same time allowing useful information to be extracted. Regular (hedge) expression patterns provide a similar feature if the values to be operated upon are strings or hedges (sequence of trees).

As an example of regular hedge expression patterns, consider the following MLlike match construct:

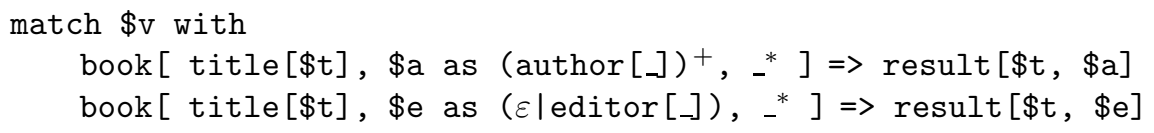

Here we have two rules. Each rule consists of a regular hedge expression pattern and an action to undertake when the pattern matches the value. Each rule is tried in turn, starting from the top, until a pattern is found for which the input hedge (in variable $\$ \mathrm{v}$ ) matches. Matching a value against a pattern consists of two parts: (1) ensuring that the input belongs to the formal language defined by the pattern; and (2) associating with every subpattern the matching part of the input. The 
obtained associations can then be used to undertake the associated action, which constructs the output.

In our example, the formal language of the first pattern consists of all (ordered) trees for which:

- the root node is labeled by book;

- the first child is labeled by title;

- this first child has one or more author nodes as right siblings; and

- those sibling nodes are followed by zero or more other nodes (the underscore denotes any tree).

If an input hedge belongs to this formal language, then variable $\$$ t should be bound to the children of the title node and variable $\$$ a should be bound to the author nodes matched by the (author []$)^{+}$subpattern. The result of this rule is constructed by creating a new node labeled result, with children $\$$ t and $\$$ a.

Likewise, the formal language of the second pattern consists of all trees for which:

- the root node is labeled by book;

- the first child is labeled by title;

- this first child is followed by an optional editor node ( $\varepsilon$ stands for the empty hedge pattern);

— which is followed by zero or more other nodes.

If an input hedge belongs to this formal language, then variable $\$$ t should be bound to the children of the title node and variable $\$$ e should be bound to the hedge matched by the ( $\varepsilon$ leditor [_] ) subpattern. The result of the second rule is constructed by creating a new node labeled result, with children $\$$ t and $\$$ e.

In general, patterns can be ambiguous, meaning that there are various ways of matching the input, resulting in multiple possible associations, and hence in multiple possible outputs.

Example 2.1. Indeed, consider the following input tree, which is depicted in Figure 1(a):

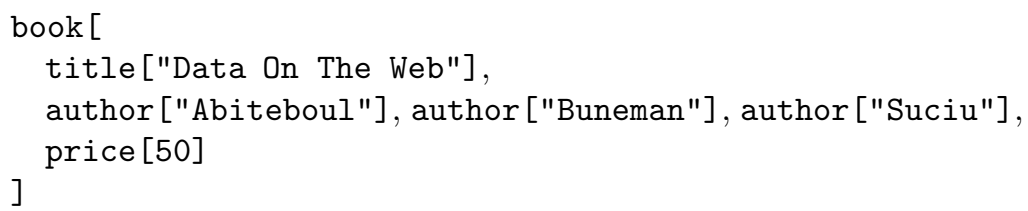

It is clear that this tree belongs to the formal language defined by the first pattern. Note, however, that there are multiple ways of "parsing" the value by the pattern. For instance, we could parse the first author node by the (author [] ] ) + subpattern, and we could parse its right siblings by the _* subpattern. Alternatively, we could parse the first two author nodes by the (author [] $)^{+}$pattern and their right siblings by the _* subpattern. Finally, we could parse all author nodes by the (author [_] $)^{+}$subpattern, and only the price node by the _* subpattern. The following table summarizes the various associations for $\$$ t and $\$$ a corresponding to these possibilities. 


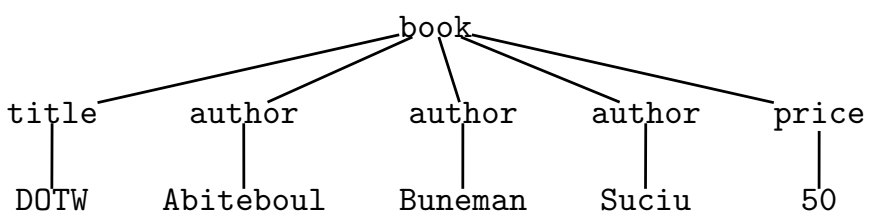

(a)

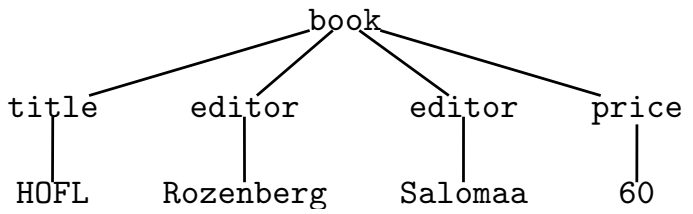

(b)

Fig. 1. (a) The input tree from Example 2.1. (b) The input tree from Example 2.2.

\begin{tabular}{l|l}
$\$ \mathrm{t}$ & $\$ \mathrm{a}$ \\
\hline "DOTW" & author ["Abiteboul"] \\
"DOTW" & $\begin{array}{l}\text { author ["Abiteboul"], author ["Buneman"] } \\
\text { "DOTW" }\end{array}$ author["Abiteboul"], author ["Buneman"], author ["Suciu"]
\end{tabular}

Note that we get a different output for each possible association.

Example 2.2. Pattern two is also ambiguous. Consider the following input tree, which is depicted in Figure 1(b):

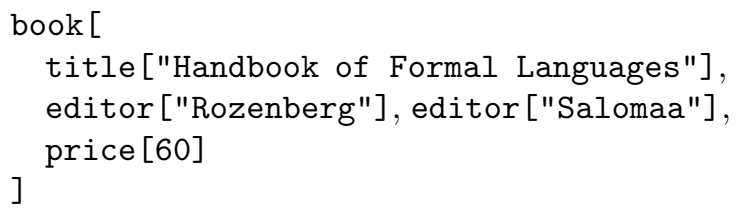

It is clear that this tree belongs to the formal language defined by the second pattern. Here we could parse the empty hedge by the ( $\varepsilon$ leditor [_] ) subpattern and the editor and price nodes by the _* pattern; or we could parse the first editor node by the ( $\varepsilon$ leditor $\left[\_\right]$) subpattern and its right siblings by _*. Note again that we get a different output for each possible association.

When patterns are used in database query languages, it is common and desirable for a pattern to have many matches in the data, and to be able to retrieve all of them [Abiteboul et al. 1997; Neumann and Seidl 1998; Buneman et al. 2000; Neven and Schwentick 2001; Murata 2001; Boag et al. 2005]. However, in generalpurpose programming using pattern matching as in ML [Ullman 1998] or Prolog [Sterling and Shapiro 1994] we normally want unique matching and a deterministic semantics. 
One approach to the latter problem would be to simply disallow ambiguity by requiring the regular expressions to be unambiguous [Book et al. 1971; Hosoya 2003]. Another, more programmer-friendly approach is to allow arbitrary regular expression patterns, but to give a disambiguation policy, which ensures a unique matching semantics. It is this approach that is taken in Perl, awk, sed, and XDuce. Amongst these applications, various disambiguation policies exist:

- The first, followed by all IEEE POSIX compliant tools, including awk and sed, consists of a single rule which states that each subpattern should match as much of the input as possible while still allowing the rest of the pattern to match [Institute of Electrical and Electronic Engineers 1992]. Subpatterns starting earlier are given priority over those starting later. We will refer to this policy as the POSIX policy.

- The second, which was informally introduced in [Hosoya 2000; Hosoya and Pierce 2002], consists of two disambiguation rules: first match and longest match. The first match rule disambiguates a disjunction pattern $\mathrm{P}_{1}+\mathrm{P}_{2}$ by giving higher priority to the first alternative $\mathrm{P}_{1}$. Moreover, disjunction distributes over concatenation. That is, when matching $w$ against $\left(\mathrm{P}_{1}+\mathrm{P}_{2}\right) \cdot \mathrm{P}_{3}, w$ should be first matched against $\mathrm{P}_{1} \cdot \mathrm{P}_{3}$ and it should only be matched against $\mathrm{P}_{2} \cdot \mathrm{P}_{3}$ when this fails. The longest match rule disambiguates the Kleene closure in patterns of the form $\mathrm{P}_{1}^{*} \cdot \mathrm{P}_{2}$ by requiring that $\mathrm{P}_{1}^{*}$ matches as much of the input as possible, still allowing the rest of the pattern to match. We will refer to this policy as the first and longest match policy.

- The third, followed by Perl, XDuce, $\mathbb{C} D u c e$, and $\lambda^{\text {re }}$ also consists of two rules: first match and greedy match [Hosoya 2000; Hosoya and Pierce 2002; Frisch et al. 2002]. The first match is the same as for the first and longest match policy. The greedy match rule disambiguates the Kleene closure in a pattern $\mathrm{P}_{1}^{*} \cdot \mathrm{P}_{2}$ by recursively rewriting it into $\left(\mathrm{P}_{1} \cdot \mathrm{P}_{1}^{*}+\varepsilon\right) \cdot \mathrm{P}_{2}$. We will refer to this policy as the XDuce policy.

Example 2.3. Consider again the matching of the input tree in Figure 1(a) against the first pattern. Because the (author [] $)^{+}$subpattern occurs before the ${ }_{-}^{*}$ subpattern, the POSIX policy requires us to match as many nodes by (author [] $)^{+}$ as possible. Hence, all author nodes are matched by this subpattern. As such, \$t is associated with "DOTW" and \$a is associated with

author ["Abiteboul"], author ["Buneman"], author ["Suciu"].

Since the (author [a] ) ${ }^{+}$subpattern is a Kleene closure, the first and longest match policy and the XDuce policy also require to match as many nodes by (author [_] ) ${ }^{+}$ as possible, resulting in the same associations.

The associations obtained under the various polices differ when the input tree of Figure 1(b) is matched against the second pattern.

Example 2.4. Indeed, since the ( $\varepsilon$ leditor [] $)$ subpattern occurs before the _* subpattern, and since matching a single tree is considered longer than matching the empty hedge, the POSIX policy will require us to match the first editor node by ( $\varepsilon$ leditor [_]), and its right siblings by _-*. Hence, under the POSIX disambiguation policy, \$t is associated with "HOFL" and $\$ \mathrm{a}$ is associated with 
editor["Salomaa"]. The first and longest policy and the XDuce policy, however, will first try to match against the $\varepsilon$ subpattern (which succeeds) before trying to match against the editor [_] subpattern. Hence, under these policies, $\$$ t is associated with "HOFL" and \$a is associated with the empty hedge. We will show the difference between the first and longest match policy and the XDuce policy in Section 6 .

In the following sections we will formally describe the matching process under all three disambiguation policies by means of the matching relation $v \in \mathrm{P} \rightsquigarrow V$, signifying that (string or hedge) input value $v$ is matched by (string or hedge) pattern $\mathrm{P}$ yielding associations $V$. We will view patterns as abstract syntax trees, and identify subpatterns by their corresponding nodes in the abstract syntax tree. This has the advantage that we do not have to mention variables explicitly in a pattern. We just have to reason about the node a variable is associated with. Hence, we can formally describe the associations $V$ as a function from nodes $n$ in $\mathrm{P}$ to subvalues of $v$ or to the special symbol $\perp$. The matching relation will be defined such that $V(n)=v^{\prime}$ if and only if the pattern rooted at node $n$ is responsible for matching the subpart $v^{\prime}$ of $v$. It is $\perp$ if the subpattern is not responsible for recognizing any subpart of $v$.

We will not concern ourselves with the efficient implementation of the matching process under the various disambiguation policies, for which we refer to the literature [Laurikari 2000; 2001; Frisch and Cardelli 2004; Frisch 2004; Levin 2003].

\subsection{Type Inference}

XDuce [Hosoya 2000; Hosoya and Pierce 2003; 2002; Hosoya et al. 2005], CDuce [Frisch et al. 2003; 2002], and $\lambda^{r e}$ [Tabuchi et al. 2002] are programming languages that can statically verify whether a transformation is type-safe. They all use regular expressions types capable of representing regular (hedge) languages to achieve this goal. Regular (hedge) languages serve as a unifying model for many schema languages [Murata et al. 2001; Neven 2002]. In order to support regular expression pattern matching XDuce, $\mathbb{C D u c e}$, and $\lambda^{r e}$ employ a type inference algorithm that calculates, for each subpattern, the set of values it can be associated with given a type for the input. The idea is to use these sets to compute the type of all constructed output values, and to check that this type is a subtype of the given output type.

In the following sections we will introduce type inference algorithms for the $\mathbb{P}$ and first and longest match disambiguation policies on strings and on hedges. We abstract away from a particular syntax of regular expression types, and use regular word and hedge languages instead. We will use $\mathcal{T}^{D}(n, \mathrm{P}, C)$ to denote the set of all values the subpattern rooted at node $n$ in $\mathrm{P}$ can be bound to under disambiguation policy $D$ when the input values all belong to the set $C$ :

$$
\mathcal{T}^{D}(n, \mathrm{P}, C):=\left\{v^{\prime} \mid \exists v \in C, v \in \mathrm{P} \rightsquigarrow V, V(n)=v^{\prime}\right\}
$$

For the superscript $D$ we will use $\mathbb{P}$ to denote the POSIX disambiguation policy, $\mathbb{F} \mathbb{L}$ to denote the first and longest match disambiguation policy, and $\mathbb{X} \mathbb{D}$ to denote the XDuce disambiguation policy. 


\section{REGULAR STRING EXPRESSION PATTERNS}

In this section we define regular string expression patterns, and provide some general notation that will be used throughout the paper.

We assume given a fixed, finite alphabet $\Sigma$ which does not contain the special symbols $\perp$ and $\square$. Elements of $\Sigma$ will be denoted by $\sigma$ and words over $\Sigma$ will be denoted by $w$ throughout the rest of this paper. The empty word is denoted by $\lambda$. A regular string expression pattern $\mathrm{P}$ is a regular expression over $\Sigma$. That is, $\mathrm{P}$ is either of the form $\varepsilon$ (with $\varepsilon$ recognizing the empty word), $\sigma$ (with $\sigma \in \Sigma$ ), $\mathrm{P}_{1}+\mathrm{P}_{2}, \mathrm{P}_{1} \cdot \mathrm{P}_{2}$, or $\mathrm{P}_{1}^{*}$, where $\mathrm{P}_{1}$ and $\mathrm{P}_{2}$ are already regular expression patterns. The language $L(\mathrm{P})$ of a pattern $\mathrm{P}$ is defined as usual. That is, $L(\varepsilon)=\{\lambda\}, L(\sigma)=\{\sigma\}$, $L\left(\mathrm{P}_{1}+\mathrm{P}_{2}\right)=L\left(\mathrm{P}_{1}\right) \cup L\left(\mathrm{P}_{2}\right), L\left(\mathrm{P}_{1} \cdot \mathrm{P}_{2}\right)$ is the concatenation of $L\left(\mathrm{P}_{1}\right)$ and $L\left(\mathrm{P}_{2}\right)$, and $L\left(\mathrm{P}_{1}^{*}\right)$ is the Kleene closure of $L\left(\mathrm{P}_{1}\right)$. Because we want to identify the subexpressions of a pattern, we abuse notation slightly and identify $\mathrm{P}$ with the partial function $\mathrm{P}:\{1,2\}^{*} \rightarrow\{*, \cdot,+, \varepsilon\} \cup \Sigma$ such that

-if $\mathrm{P}=\varepsilon$ then $\operatorname{dom}(\mathrm{P})=\{\lambda\}$ and $\mathrm{P}(\lambda)=\varepsilon$;

-if $\mathrm{P}=\sigma$ with $\sigma \in \Sigma$ then $\operatorname{dom}(\mathrm{P})=\{\lambda\}$ and $\mathrm{P}(\lambda)=\sigma$;

-if $\mathrm{P}=\mathrm{P}_{1}+\mathrm{P}_{2}$ then $\operatorname{dom}(\mathrm{P})=\{\lambda\} \cup\left\{1 n \mid n \in \operatorname{dom}\left(\mathrm{P}_{1}\right)\right\} \cup\left\{2 n \mid n \in \operatorname{dom}\left(\mathrm{P}_{2}\right)\right\}$ with $\mathrm{P}(\lambda)=+, \mathrm{P}(1 n)=\mathrm{P}_{1}(n)$, and $\mathrm{P}(2 n)=\mathrm{P}_{2}(n)$;

-if $\mathrm{P}=\mathrm{P}_{1} \cdot \mathrm{P}_{2}$ then $\operatorname{dom}(\mathrm{P})=\{\lambda\} \cup\left\{1 n \mid n \in \operatorname{dom}\left(\mathrm{P}_{1}\right)\right\} \cup\left\{2 n \mid n \in \operatorname{dom}\left(\mathrm{P}_{2}\right)\right\}$ with $\mathrm{P}(\lambda)=\cdot, \mathrm{P}(1 n)=\mathrm{P}_{1}(n)$, and $\mathrm{P}(2 n)=\mathrm{P}_{2}(n) ;$ and

-if $\mathrm{P}=\mathrm{P}_{1}^{*}$ then $\operatorname{dom}(\mathrm{P})=\{\lambda\} \cup\left\{1 n \mid n \in \operatorname{dom}\left(\mathrm{P}_{1}\right)\right\}, \mathrm{P}(\lambda)=*$, and $\mathrm{P}(1 n)=\mathrm{P}_{1}(n)$.

Intuitively, the function view of a pattern describes the abstract syntax tree of its regular expression, as shown in Figure 2. In general, an expression can have multiple parse trees. We therefore assume the usual precedence of operators in the previous definition: ${ }^{*}$ binds tighter than $\cdot$, which has a higher precedence than + . Furthermore, $\cdot$ and + are assumed to be right-associative. Elements of $\{1,2\}^{*}$ are called nodes and will be denoted by $n, m$, and their subscripted versions. We write $|\mathrm{P}|$ for the number of nodes of P. Intuitively, nodes are used to identify subexpressions.

Since subpatterns inside a Kleene closure can match multiple subwords of an input word, we will not compute associations for such subpatterns. Therefore, a node $n \in \operatorname{dom}(\mathrm{P})$ is a bindable node of $\mathrm{P}$ if it does not have an ancestor labeled with $*$. The set of bindable nodes of $\mathrm{P}$ is denoted by $b n(\mathrm{P})$.

As was already noted in Section 2, the matching process for a given disambiguation strategy is formally described by the matching relation $w \in \mathrm{P} \rightsquigarrow V$, signifying that $w$ is matched by $\mathrm{P}$ yielding associations $V$. Here, $V$ is a function from $b n(\mathrm{P})$ to subwords of $w$ or to the special symbol $\perp$. The matching relation will be defined such that $V(n)=w^{\prime}$ if and only if the pattern rooted at node $n$ is responsible for matching the subword $w^{\prime}$ under the considered disambiguation policy. It is $\perp$ if the subpattern is not responsible for recognizing any subword of $w$.

Example 3.1. As we will further illustrate in Example 4.1, matching the word $a b$ against the pattern $(a+a \cdot b) \cdot(b+\varepsilon)$ of Figure 2(a) under the POSIX disambiguation 


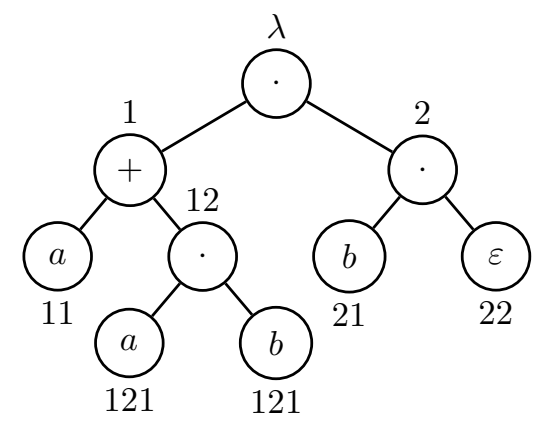

(a)

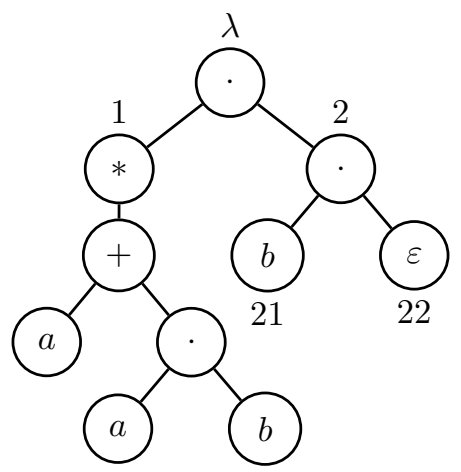

(b)

Fig. 2. The abstract syntax tree representation of $(a+a \cdot b) \cdot(b+\varepsilon)$ (left) and $(a+a \cdot b)^{*} \cdot(b+\varepsilon)$ (right). The bindable nodes have their addresses annotated.

strategy yields the associations $V$ where

$$
\begin{array}{rlrlrl}
V(\lambda) & =a b & V(1) & =a b & V(11) & =\perp \\
V(12) & =a b & V(121) & =a & V(122) & =b \\
V(2) & =\lambda & V(21) & =\perp & V(22) & =\lambda .
\end{array}
$$

On the other hand, matching $a b$ against this pattern under the first and longest match disambiguation policy yields the associations $V^{\prime}$ where

$$
\begin{aligned}
V^{\prime}(\lambda) & =a b & V^{\prime}(1) & =a & V^{\prime}(11) & =a \\
V^{\prime}(12) & =\perp & V^{\prime}(121) & =\perp & V^{\prime}(122) & =\perp \\
V^{\prime}(2) & =b & V^{\prime}(21) & =b & V^{\prime}(22) & =\perp,
\end{aligned}
$$

as we will further illustrate in Example 6.1.

To simplify the definition of matching relations we introduce the following notation. Let $V_{1}$ and $V_{2}$ be associations, and let $\mathrm{P}_{1}$ and $\mathrm{P}_{2}$ be patterns. We write $[\lambda \rightarrow w]$ to denote the function with domain $\{\lambda\}$ for which

$$
[\lambda \rightarrow w](\lambda)=w .
$$

We write $V_{1}+\mathrm{P}_{2}$ to denote the function for which

$$
\left(V_{1}+\mathrm{P}_{2}\right)(n)= \begin{cases}V_{1}(1) & \text { if } n=\lambda \\ V_{1}(m) & \text { if } n=1 m, m \in \operatorname{dom}\left(V_{1}\right) \\ \perp & \text { if } n=2 m, m \in \operatorname{dom}\left(\mathrm{P}_{2}\right) .\end{cases}
$$

We define $\mathrm{P}_{1}+V_{2}$ similarly:

$$
\left(\mathrm{P}_{1}+V_{2}\right)(n)= \begin{cases}V_{2}(1) & \text { if } n=\lambda ; \\ V_{2}(m) & \text { if } n=2 m, m \in \operatorname{dom}\left(V_{2}\right) \\ \perp & \text { if } n=1 m, m \in \operatorname{dom}\left(\mathrm{P}_{1}\right) .\end{cases}
$$

ACM Transactions on Programming Languages and Systems, Vol. TBD, No. TDB, Month Year. 
Finally, we denote by $V_{1} \cdot V_{2}$ the function such that

$$
\left(V_{1} \cdot V_{2}\right)(n)= \begin{cases}V_{1}(\lambda) \cdot V_{2}(\lambda) & \text { if } n=\lambda, V_{1}(\lambda) \neq \perp, V_{2}(\lambda) \neq \perp ; \\ \perp & \text { if } n=\lambda \text { and }\left(V_{1}(\lambda)=\perp \text { or } V_{2}(\lambda)=\perp\right) ; \\ V_{1}(m) & n=1 m, m \in \operatorname{dom}\left(V_{1}\right) \\ V_{2}(m) & n=2 m, m \in \operatorname{dom}\left(V_{2}\right) .\end{cases}
$$

Example 3.2. If $V_{1}$ is the association function with domain $\{\lambda, 1,2\}$ such that

$$
V_{1}(\lambda)=a b \quad V_{1}(1)=a \quad V_{1}(2)=b,
$$

then $a+V_{1}$ is the association function $W$ with domain $\{\lambda, 1,2,21,22\}$ such that

$$
\begin{array}{rlrl}
W(\lambda) & =a b & W(1) & =\perp \\
W(21) & =a & W(22) & =b .
\end{array} \quad W(2)=a b
$$

Furthermore, if $V_{2}$ is the association function with domain $\{\lambda, 1,2\}$ such that

$$
V_{2}(\lambda)=\lambda \quad V_{2}(1)=\perp \quad V_{2}(2)=\lambda,
$$

then $\left(a+V_{1}\right) \cdot V_{2}$ is the association function $V$ from Example 3.1. I.e., it is the association obtained by matching the word $a b$ against pattern $\mathrm{P}$ from Figure 2(a) under the POSIX disambiguation policy.

\section{MATCHING UNDER THE POSIX POLICY}

As shown in Section 2, patterns can be ambiguous, meaning that there are various ways of matching an input word. In this section we formally introduce the POSIX disambiguation policy, employed by all IEEE POSIX standard compliant regular expression tools like awk, sed, ... It is easy to formalize and the techniques for its associated type inference algorithm, as developed in the next section, serve as a warmup for that of the first and longest match policy treated in the second half of this paper.

The POSIX disambiguation policy can be expressed as follows [Institute of Electrical and Electronic Engineers 1992; Laurikari 2001]:

Subpatterns should match the longest possible substrings, where subpatterns that start earlier in the regular expression take priority over ones starting later. Hence, higher-level subpatterns take priority over their lower-level component subpatterns. Matching an empty string is considered longer than no match at all.

Let us clarify this rule with an example.

Example 4.1. Consider the matching of $a b$ against the pattern $(a+a \cdot b) \cdot(b+\varepsilon)$ of Figure 2(a). Then the whole pattern matches $a b$. Because subpattern $(a+a \cdot b)$ starts earlier than $(b+\varepsilon)$, it should match as much of the input string as possible, still allowing the whole pattern to match. Hence, $(a+a \cdot b)$ matches $a b$ and $(b+\varepsilon)$ matches $\lambda$.

The matching relation $w \in \mathrm{P} \rightsquigarrow V$ under the POSIX policy is formally defined in Figure 3. Rules EMPTY and LAB are axioms allowing to match the empty sequence and a single symbol respectively. Rule KLEENE allows matching a word against a 


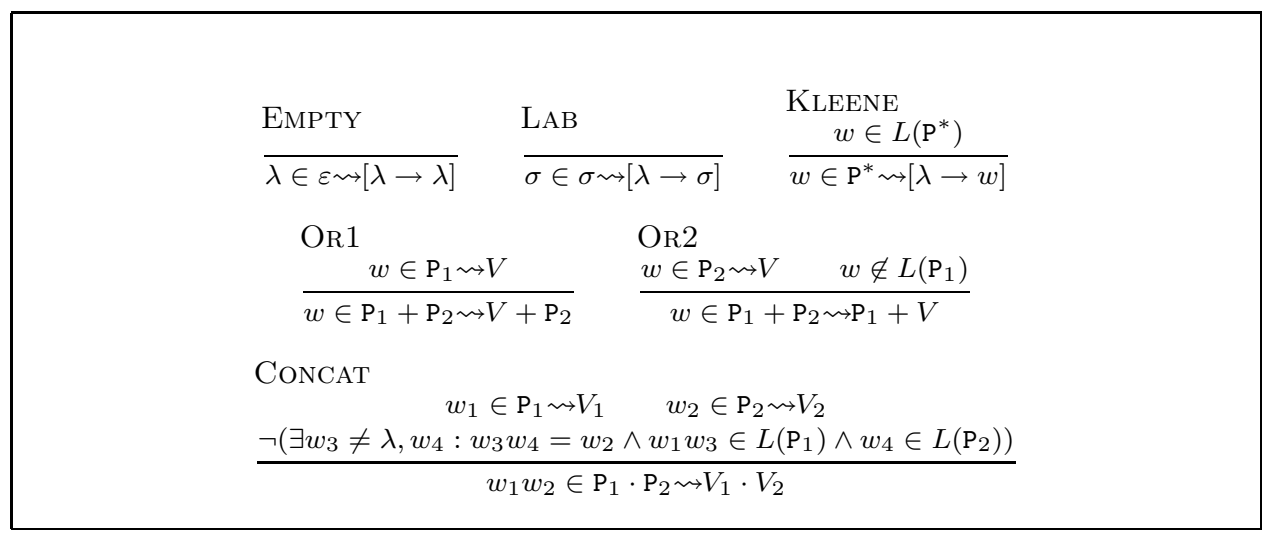

Fig. 3. The matching relation $w \in \mathrm{P} \rightsquigarrow V$ under the POSIX disambiguation policy.

Kleene closure pattern. Note that the resulting association function only provides an association for $\lambda$, which is the only bindable node of $\mathrm{P}^{*}$. For a disjunction $\mathrm{P}_{1}+\mathrm{P}_{2}$, the POSIX disambiguation policy specifies that the whole pattern should match the longest possible substring. In order for the match to succeed, this would have to be the whole input word. Furthermore, since $\mathrm{P}_{1}$ starts earlier than $\mathrm{P}_{2}, \mathrm{P}_{1}$ is to be given precedence. Consequently, when matching $w$ against $\mathrm{P}_{1}+\mathrm{P}_{2}$, we should always try to match $w$ to $\mathrm{P}_{1}$ first. This is expressed in rules OR 1 and OR2, where Or2 can only be used if Or1 fails. Rule CONCAT specifies that in a concatenation $\mathrm{P}_{1} \cdot \mathrm{P}_{2}$, pattern $\mathrm{P}_{1}$ should match as much as possible (since it occurs earlier), still allowing the entire pattern to match.

THEOREM 4.2. The matching relation of Figure 3 is well defined:

(1) The matching relation is semantically correct: $w \in P \rightsquigarrow V$ iff $w \in L(P)$, and,

(2) The matching relation is unique: if $w \in P \rightsquigarrow V$ and $w \in P \rightsquigarrow W$ then $V=W$.

Proof. (1). The "if" direction can be proved by a straightforward induction on P. The "only if" direction can be proved by a straightforward induction on the matching derivation.

(2). By a straightforward induction on the matching derivation of $w \in \mathrm{P} \rightsquigarrow V$, with a case analysis on the last rule used.

Example 4.3. The following is the matching derivation of $a b$ against $(a+a \cdot b)$. $(b+\varepsilon):$

\begin{tabular}{|c|c|c|c|}
\hline LAB & LAB & \multirow{4}{*}{$\begin{array}{l}\text { CONCAT } \\
\text { COR2 }\end{array}$} & \multirow{4}{*}{$\begin{array}{l}\text { ЕмРту } \\
\frac{\lambda \in \varepsilon \rightsquigarrow V_{3}:=[\lambda \rightarrow \lambda]}{\lambda \in(b+\varepsilon) \rightsquigarrow b+V_{3}}\end{array}$} \\
\hline$\overline{a \in a \rightsquigarrow V_{1}:=[\lambda \rightarrow a]}$ & $\overline{b \in b \rightsquigarrow V_{2}:=[\lambda \rightarrow b]}$ & & \\
\hline \multicolumn{2}{|c|}{$a b \in a \cdot b \leadsto V_{1} \cdot V_{2}$} & & \\
\hline$a b \in(a+a$. & $a+\left(V_{1} \cdot V_{2}\right)$ & & \\
\hline
\end{tabular}


It is easily seen that the obtained association function $\left(a+\left(V_{1} \cdot V_{2}\right)\right) \cdot\left(b+V_{3}\right)$ equals the association function $V$ from Example 3.1. For example,

$$
\left(\left(a+\left(V_{1} \cdot V_{2}\right)\right) \cdot\left(b+V_{3}\right)\right)(1)=\left(a+\left(V_{1} \cdot V_{2}\right)\right)(\lambda)=V_{1}(\lambda) \cdot V_{2}(\lambda)=a b=V(1) .
$$

Likewise:

$$
\left(\left(a+\left(V_{1} \cdot V_{2}\right)\right) \cdot\left(b+V_{3}\right)\right)(21)=\left(b+V_{3}\right)(1)=\perp=V(21) .
$$

We note that we cannot match $a$ by $(a+a \cdot b)$ and $b$ by $(b+\varepsilon)$. Indeed, although it is possible to derive $a \in(a+a \cdot b) \rightsquigarrow W_{1}$ and $b \in b+\varepsilon \rightsquigarrow W_{2}$ for some associations $W_{1}$ and $W_{2}$, the third premise of rule ConcAT will disable us to conclude $a b \in$ $(a+a \cdot b) \cdot(b+\varepsilon) \rightsquigarrow W_{1} \cdot W_{2}$. Indeed, since $a b \in L(a+a \cdot b)$ and $\lambda \in L(b+\varepsilon)$ there exists a longer match.

\section{TYPE INFERENCE UNDER THE POSIX POLICY}

The matching process described in the previous section is used in UNIX tools like sed and awk [Dougherty and Robbins 1996]. Solving its regular type inference problem can be seen as a first step towards making transformations in these languages type safe. The main result of this section can be stated as follows:

TheOREM 5.1. If $C$ is a regular language then $\mathcal{T}^{\mathbb{P}}(m, P, C)$ is also regular, and can be effectively computed.

\subsection{The Algorithm}

Let us first introduce the algorithm by informal reasoning. We will formally prove its correctness later.

We observe that the type of the root node $\lambda$ is exactly the set of words in $C$ that can be matched by P. Indeed, if $w$ is successfully matched by $\mathrm{P}$ then $\lambda$ is associated to $w$ itself. If $m \neq \lambda$, then $\mathrm{P}$ is of the form $\mathrm{P}_{1}+\mathrm{P}_{2}$ or $\mathrm{P}_{1} \cdot \mathrm{P}_{2}$, since all other patterns contain only one bindable node: $\lambda$.

If $\mathrm{P}=\mathrm{P}_{1}+\mathrm{P}_{2}$ then we observe that words can only be associated to subpatterns of $\mathrm{P}_{1}$ if they are subwords of some word in $C$ matched by $\mathrm{P}_{1}$. Hence, if $m=1 n$ then we can calculate $\mathcal{T}^{\mathbb{P}}(1 n, \mathrm{P}, C)$ simply by calculating $\mathcal{T}^{\mathbb{P}}\left(n, \mathrm{P}_{1}, C\right)$. Similarly, words can only be associated to subpatterns of $\mathrm{P}_{2}$ if they are subwords of some word in $C$ matched by $\mathrm{P}_{2}$. We must take care however, since this word must not be matched against $P_{1}$ because of the precedence of $P_{1}$ over $P_{2}$ in $P$. Hence, we can calculate $\mathcal{T}^{\mathbb{P}}(2 n, \mathrm{P}, C)$ by calculating $\mathcal{T}^{\mathbb{P}}\left(n, \mathrm{P}_{2}, C-L\left(\mathrm{P}_{1}\right)\right)$.

If $\mathrm{P}=\mathrm{P}_{1} \cdot \mathrm{P}_{2}$ then we observe that words can only be associated to subpatterns of $\mathrm{P}_{1}$ if they are subwords of a word $w_{1}$ matched by $\mathrm{P}_{1}$, for which there exists some $w_{2}$ matched by $\mathrm{P}_{2}$ such that $w_{1} w_{2} \in C$ and such that $w_{1}$ really is the longest possible prefix of $w_{1} w_{2}$ that can be matched by $\mathrm{P}_{1}$, still allowing the corresponding suffix to be matched by $\mathrm{P}_{2}$. Formally this means that we cannot break $w_{2}$ in $w_{3} \neq \lambda$ and $w_{4}$ with $w_{1} w_{3} \in L\left(\mathrm{P}_{1}\right)$ and $w_{4} \in L\left(\mathrm{P}_{2}\right)$. Let us define the left breaking of $C$ by languages $L_{1}$ and $L_{2}$, denoted by $\operatorname{lbreak}\left(C, L_{1}, L_{2}\right)$, to be exactly the set of such words $w_{1}$ :

$$
\begin{aligned}
\operatorname{lbreak}\left(C, L_{1}, L_{2}\right):=\left\{w_{1} \in L_{1} \mid \exists w_{2} \in L_{2}: w_{1} w_{2} \in C\right. & \\
& \left.\wedge \neg\left(\exists w_{3} \neq \lambda, w_{4}: w_{3} w_{4}=w_{2} \wedge w_{1} w_{3} \in L_{1} \wedge w_{4} \in L_{2}\right)\right\} .
\end{aligned}
$$




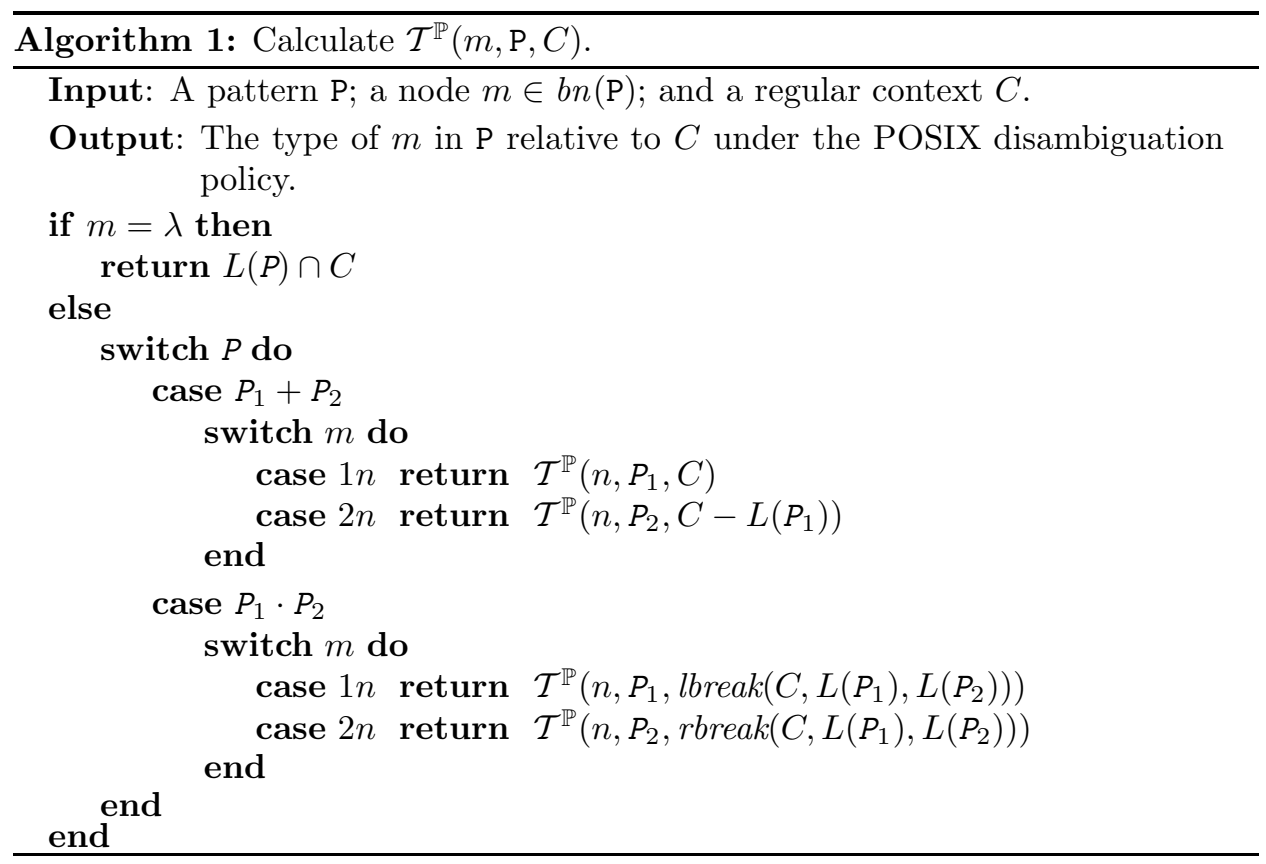

Then $\mathcal{T}^{\mathbb{P}}(1 n, \mathrm{P}, C)$ equals $\mathcal{T}^{\mathbb{P}}\left(n, \mathrm{P}_{1}\right.$, lbreak $\left.\left(C, L\left(\mathrm{P}_{1}\right), L\left(\mathrm{P}_{2}\right)\right)\right)$. Similarly, words can only be associated to subpatterns of $\mathrm{P}_{2}$ in $\mathrm{P}_{1} \cdot \mathrm{P}_{2}$ if they are subwords of a word $w_{2}$ matched by $\mathrm{P}_{2}$ for which there exists some $w_{1}$ matched by $\mathrm{P}_{1}$ such that $w_{1} w_{2} \in C$ and such that $w_{1}$ really is the longest possible prefix of $w_{1} w_{2}$ matched by $\mathrm{P}_{1}$, still allowing the corresponding suffix to be matched by $\mathrm{P}_{2}$. The formal requirement is the same as before. Let us define the right breaking of $C$ by languages $L_{1}$ and $L_{2}$, denoted as $\operatorname{rbreak}\left(C, L_{1}, L_{2}\right)$ to be exactly the set of such words $w_{2}$ :

$$
\begin{aligned}
\operatorname{rbreak}\left(C, L_{1}, L_{2}\right):= & \left\{w_{2} \in L_{2} \mid \exists w_{1} \in L_{1}: w_{1} w_{2} \in C\right. \\
& \left.\wedge \neg\left(\exists w_{3} \neq \lambda, w_{4}: w_{3} w_{4}=w_{2} \wedge w_{1} w_{3} \in L_{1} \wedge w_{4} \in L_{2}\right)\right\} .
\end{aligned}
$$

Then $\mathcal{T}^{\mathbb{P}}(2 n, \mathrm{P}, C)$ equals $\mathcal{T}^{\mathbb{P}}\left(n, \mathrm{P}_{2}\right.$, rbreak $\left.\left(C, L\left(\mathrm{P}_{1}\right), L\left(\mathrm{P}_{2}\right)\right)\right)$.

As we will show below, the sets $\operatorname{lbreak}\left(C, L_{1}, L_{2}\right)$ and $\operatorname{rbreak}\left(C, L_{1}, L_{2}\right)$ are regular and can effectively be computed if $C, L_{1}$, and $L_{2}$ are regular. The type inference algorithm for the POSIX matching policy is then shown in Algorithm 1. It is welldefined if we start with a regular set $C$, since all used operations can effectively be computed for regular languages. Moreover, the algorithm is terminating since the depth of the nodes to be calculated get smaller upon each recursive call.

\subsection{Computing the breaking of $C$}

In order for Algorithm 1 to make any sense, we need a way to calculate the sets lbreak $\left(C, L\left(\mathrm{P}_{1}\right), L\left(\mathrm{P}_{2}\right)\right)$ and $\operatorname{rbreak}\left(C, L\left(\mathrm{P}_{1}\right), L\left(\mathrm{P}_{2}\right)\right)$. We first need some auxiliary notions in order to develop a computation strategy.

The left quotient of language $L$ by language $K$, denoted by $K \backslash L$, is defined as $\{s \mid \exists p \in K: p s \in L\}$. The right quotient of $L$ by $K$, denoted by $L / K$, is defined 
as $\{p \mid \exists s \in K: p s \in L\}$. It is well-known that regular languages are closed under both quotients [Hopcroft and Ullman 1979].

Let $\square$ be a special symbol not in $\Sigma$. Let us write $\pi(w)$ for the word $w_{1} w_{2} \ldots w_{n}$ if $w=w_{1} \square w_{2} \square \cdots \square w_{n}$. It is easy to see that if $L$ is a regular language, then so is $\pi^{-1}(L)=\left\{w_{1} \square w_{2} \square \cdots \square w_{n} \mid w_{1} w_{2} \ldots w_{n} \in L\right\}$ (modify a DFA for $L$ to allow reading the letter $\square$, which is then ignored).

The breaking of $C$ by $L_{1}$ and $L_{2}$, denoted by $\operatorname{break}\left(C, L_{1}, L_{2}\right)$, is defined as:

$$
\begin{aligned}
& \operatorname{break}\left(C, L_{1}, L_{2}\right):=\left\{w_{1} \square w_{2} \mid w_{1} w_{2} \in C \wedge w_{1} \in L_{1} \wedge w_{2} \in L_{2}\right. \\
&\left.\wedge \neg\left(\exists w_{3} \neq \lambda, w_{4}: w_{3} w_{4}=w_{2} \wedge w_{1} w_{3} \in L_{1} \wedge w_{4} \in L_{2}\right)\right\} .
\end{aligned}
$$

Lemma 5.2. If $C, L_{1}$, and $L_{2}$ are regular, then so are the breaking, left breaking and right breaking of $C$ by $L_{1}$ and $L_{2}$. More specifically, with $A$ abbreviating the language $\pi^{-1}\left(L_{1}\right)-\left(L_{1} \cdot\{\square\}\right)$, we have:

$-\operatorname{break}\left(C, L_{1}, L_{2}\right)=\pi^{-1}(C) \cap\left(\left(L_{1} \cdot\{\square\} \cdot L_{2}\right)-A \cdot L_{2}\right)$,

- $\operatorname{lbreak}\left(C, L_{1}, L_{2}\right)=\operatorname{break}\left(C, L_{1}, L_{2}\right) /\left(\{\square\} \cdot L_{2}\right)$, and

$-\operatorname{rbreak}\left(C, L_{1}, L_{2}\right)=\left(L_{1} \cdot\{\square\}\right) \backslash \operatorname{break}\left(C, L_{1}, L_{2}\right)$.

Proof. By definition, $\left(L_{1} \cdot\{\square\} \cdot L_{2}\right)-A \cdot L_{2}$ equals

$$
\left\{w_{1} \square w_{2} \mid w_{1} \in L_{1} \wedge w_{2} \in L_{2} \wedge \neg\left(\exists v_{1}, v_{2}: v_{1} v_{2}=w_{1} \square w_{2} \wedge v_{1} \in A \wedge v_{2} \in L_{2}\right)\right\} .
$$

Or, more elaborately,

$$
\begin{aligned}
& \left\{w_{1} \square w_{2} \mid w_{1} \in L_{1} \wedge w_{2} \in L_{2}\right. \\
& \left.\quad \wedge \neg\left(\exists v_{1}, v_{2}: v_{1} v_{2}=w_{1} \square w_{2} \wedge \pi\left(v_{1}\right) \in L_{1} \wedge\left(\forall p \in L_{1}: v_{1} \neq p \square\right) \wedge v_{2} \in L_{2}\right)\right\} .
\end{aligned}
$$

We show that this equals

$$
\begin{aligned}
\left\{w_{1} \square w_{2} \mid w_{1} \in L_{1} \wedge\right. & w_{2} \in L_{2} \\
& \left.\wedge \neg\left(\exists w_{3} \neq \lambda, w_{4}: w_{2}=w_{3} w_{4} \wedge w_{1} w_{3} \in L_{1} \wedge w_{4} \in L_{2}\right)\right\} .
\end{aligned}
$$

We can see this as follows. Suppose that $w_{1} \square w_{2}$ is in the upper set and suppose that there do exist $w_{3}$ and $w_{4}$ such that $w_{2}=w_{3} w_{4}, w_{3} \neq \lambda, w_{1} w_{3} \in L_{1}$ and $w_{4} \in L_{2}$. Then take $v_{1}=w_{1} \square w_{3}$ and $v_{2}=w_{4}$ to see that $w_{1} \square w_{2}$ cannot be in the upper set, a contradiction. On the other hand, suppose $w_{1} \square w_{2}$ is in the lower set and suppose that there do exist $v_{1}$ and $v_{2}$ such that $v_{1} v_{2}=w_{1} \square w_{2}, \pi\left(v_{1}\right) \in L_{1}$, $\forall p \in L_{1}: v_{1} \neq p \square$ and $v_{2} \in L_{2}$. Since $v_{2} \in L_{2}$ and since $L_{2}$ is a language over $\Sigma$, $v_{2}$ cannot contain the symbol $\square$. Since $v_{1} v_{2}=w_{1} \square w_{2}, v_{2}$ must be a suffix of $w_{2}$. Hence, we can divide $w_{2}$ in $w_{3}$ and $w_{4}$ such that $v_{1}=w_{1} \square w_{3}$ and $v_{2}=w_{4}$. Since $v_{1} \neq p \square$ for any $p, w_{3}$ must be different from $\lambda$. Moreover, we immediately have $w_{1} w_{3}=\pi\left(v_{1}\right) \in L_{1}$ and $w_{4}=v_{2} \in L_{2}$, which gives us a contradiction.

As a consequence, $\pi^{-1}(C) \cap\left(\left(L_{1} \cdot\{\square\} \cdot L_{2}\right)-A \cdot L_{2}\right)$ must equal

$$
\begin{aligned}
\left\{w_{1} \square w_{2} \mid w_{1} w_{2}\right. & \in C \wedge w_{1} \in L_{1} \wedge w_{2} \in L_{2} \\
& \left.\wedge \neg\left(\exists w_{3}, w_{4}: w_{3} \neq \lambda \wedge w_{3} w_{4}=w_{2} \wedge w_{1} w_{3} \in L_{1} \wedge w_{4} \in L_{2}\right)\right\} .
\end{aligned}
$$

Hence, $\pi^{-1}(C) \cap\left(\left(L_{1} \cdot\{\square\} \cdot L_{2}\right)-A \cdot L_{2}\right)=\operatorname{break}\left(C, L_{1}, L_{2}\right)$, as desired. With $\phi$ abbreviating $\neg\left(\exists w_{3} \neq \lambda, w_{4}: w_{3} w_{4}=w_{2} \wedge w_{1} w_{3} \in L_{1} \wedge w_{4} \in L_{2}\right)$ we obtain the 
other two desired equalities:

$$
\begin{aligned}
\operatorname{break}\left(C, L_{1}, L_{2}\right) /\left(\{\square\} \cdot L_{2}\right) & =\left\{w_{1} \mid \exists w_{2} \in L_{2}: w_{1} \square w_{2} \in \operatorname{break}\left(C, L_{1}, L_{2}\right)\right\} \\
& =\left\{w_{1} \mid \exists w_{2} \in L_{2}: w_{1} w_{2} \in C \wedge w_{1} \in L_{1} \wedge \phi\right\} \\
& =\operatorname{lbreak}\left(C, L_{1}, L_{2}\right) \\
\left(L_{1} \cdot\{\square\}\right) \backslash \operatorname{break}\left(C, L_{1}, L_{2}\right) & =\left\{w_{2} \mid \exists w_{1} \in L_{1}: w_{1} \square w_{2} \in \operatorname{break}\left(C, L_{1}, L_{2}\right)\right\} \\
& =\left\{w_{2} \mid \exists w_{1} \in L_{1}: w_{1} w_{2} \in C \wedge w_{2} \in L_{2} \wedge \phi\right\} \\
& =\operatorname{rbreak}\left(C, L_{1}, L_{2}\right)
\end{aligned}
$$

\subsection{Proof of Correctness}

In this section we formally prove the correctness of Algorithm 1, thereby also proving Theorem 5.1.

LEMMA 5.3. If $w \in P \rightsquigarrow V$ then $V(\lambda)=w$.

PROOF. By a straightforward induction on the matching derivation.

Proposition 5.4. $\mathcal{T}^{\mathbb{P}}(\lambda, P, C)=L(P) \cap C$ for any pattern $P$.

PRoOF. By Lemma 5.3 and Theorem 4.2 it readily follows:

$$
\begin{aligned}
w \in \mathcal{T}^{\mathbb{P}}(\lambda, \mathrm{P}, C) & \Leftrightarrow \exists w^{\prime} \in C: w^{\prime} \in \mathrm{P} \rightsquigarrow V \wedge V(\lambda)=w \\
& \Leftrightarrow w \in C \wedge w \in \mathrm{P} \rightsquigarrow V \\
& \Leftrightarrow w \in C \wedge w \in L(\mathrm{P})
\end{aligned}
$$

Proposition 5.5. For $P=P_{1}+P_{2}$, the following equalities hold:

(1) $\mathcal{T}^{\mathbb{P}}(1 n, P, C)=\mathcal{T}^{\mathbb{P}}\left(n, P_{1}, C\right)$

(2) $\mathcal{T}^{\mathbb{P}}(2 n, P, C)=\mathcal{T}^{\mathbb{P}}\left(n, P_{2}, C-L\left(P_{1}\right)\right)$

PROOF. We first note that the top of a derivation for $w^{\prime} \in \mathrm{P} \rightsquigarrow V$ has two possible forms:

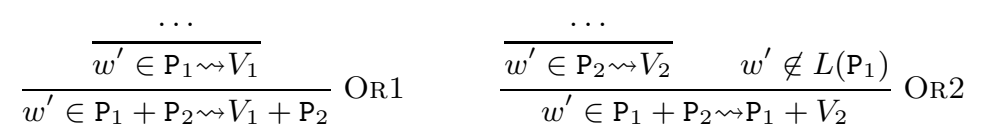

Note that, if $w^{\prime} \in \mathrm{P} \rightsquigarrow V$ and $V(1) \neq \perp$, then the derivation of $w^{\prime} \in \mathrm{P} \rightsquigarrow V$ must be of the left form. Indeed, $V(1)=\perp$ for derivations of the right form. It is then easy to see that (1) holds:

$$
\begin{aligned}
w \in \mathcal{T}^{\mathbb{P}}(1 n, \mathrm{P}, C) & \Leftrightarrow \exists w^{\prime} \in C: w^{\prime} \in \mathrm{P} \rightsquigarrow V \wedge V(1 n)=w \\
& \Leftrightarrow \exists w^{\prime} \in C: w^{\prime} \in \mathrm{P} \rightsquigarrow\left(V_{1}+\mathrm{P}_{2}\right) \wedge V_{1}(n)=w \\
& \Leftrightarrow \exists w^{\prime} \in C: w^{\prime} \in \mathrm{P}_{1} \rightsquigarrow V_{1} \wedge V_{1}(n)=w \\
& \Leftrightarrow w \in \mathcal{T}^{\mathbb{P}}\left(n, \mathrm{P}_{1}, C\right)
\end{aligned}
$$


Likewise, if $w^{\prime} \in \mathrm{P} \rightsquigarrow V$ and $V(2) \neq \perp$, then the derivation of $w^{\prime} \in \mathrm{P} \rightsquigarrow V$ must be of the right form. Indeed, $V(2)=\perp$ for derivations of the left form. It is then easy to see that (2) also holds:

$$
\begin{aligned}
w \in \mathcal{T}^{\mathbb{P}}(2 n, \mathrm{P}, C) & \Leftrightarrow \exists w^{\prime} \in C: w^{\prime} \in \mathrm{P} \rightsquigarrow V \wedge V(2 n)=w \\
& \Leftrightarrow \exists w^{\prime} \in C: w^{\prime} \in \mathrm{P} \rightsquigarrow\left(\mathrm{P}_{1}+V_{2}\right) \wedge V_{2}(n)=w \\
& \Leftrightarrow \exists w^{\prime} \in C: w^{\prime} \notin L\left(\mathrm{P}_{1}\right) \wedge w^{\prime} \in \mathrm{P}_{2} \rightsquigarrow V_{2} \wedge V_{2}(n)=w \\
& \Leftrightarrow w \in \mathcal{T}^{\mathbb{P}}\left(n, \mathrm{P}_{2}, C-L\left(\mathrm{P}_{1}\right)\right)
\end{aligned}
$$

Proposition 5.6. When $P=P_{1} \cdot P_{2}$ the following equalities hold:

(1) $\mathcal{T}^{\mathbb{P}}(1 n, P, C)=\mathcal{T}^{\mathbb{P}}\left(n, P_{1}\right.$, lbreak $\left.\left(C, L\left(P_{1}\right), L\left(P_{2}\right)\right)\right)$

(2) $\mathcal{T}^{\mathbb{P}}(2 n, P, C)=\mathcal{T}^{\mathbb{P}}\left(n, P_{2}, \operatorname{rbreak}\left(C, L\left(P_{1}\right), L\left(P_{2}\right)\right)\right)$

Proof. Note that for any derivation of $w^{\prime} \in \mathrm{P} \rightsquigarrow V$, the top must look like:

$$
\begin{gathered}
\frac{\cdots}{w_{1} \in \mathrm{P}_{1} \rightsquigarrow V_{1}} \quad \frac{\cdots}{w_{2} \in \mathrm{P}_{2} \rightsquigarrow V_{2}} \\
\frac{\neg\left(\exists w_{3} \neq \lambda, w_{4}: w_{2}=w_{3} w_{4} \wedge w_{1} w_{3} \in L\left(\mathrm{P}_{1}\right) \wedge w_{4} \in L\left(\mathrm{P}_{2}\right)\right)}{w^{\prime}=w_{1} w_{2} \in \mathrm{P} \rightsquigarrow V=V_{1} \cdot V_{2}} \text { CONCAT }
\end{gathered}
$$

Using Theorem 4.2, equality (1) then readily follows:

$$
\begin{aligned}
w \in & \mathcal{T}^{\mathbb{P}}(1 n, \mathrm{P}, C) \\
\Leftrightarrow & \exists w^{\prime} \in C: w^{\prime} \in \mathrm{P} \rightsquigarrow V \wedge V(1 n)=w \\
\Leftrightarrow & \exists w_{1}, w_{2}: w_{1} w_{2} \in C \wedge w_{1} \in \mathrm{P}_{1} \rightsquigarrow V_{1} \wedge w_{2} \in \mathrm{P}_{2} \rightsquigarrow V_{2} \wedge V_{1}(n)=w \\
& \wedge \neg\left(\exists w_{3} \neq \lambda, w_{4}: w_{3} w_{4}=w_{2} \wedge w_{1} w_{3} \in L\left(\mathrm{P}_{1}\right) \wedge w_{4} \in L\left(\mathrm{P}_{2}\right)\right) \\
\Leftrightarrow & \exists w_{1} \in L\left(\mathrm{P}_{1}\right), w_{2} \in L\left(\mathrm{P}_{2}\right): w_{1} w_{2} \in C \wedge w_{1} \in \mathrm{P}_{1} \rightsquigarrow V_{1} \wedge V_{1}(n)=w \\
& \wedge \neg\left(\exists w_{3} \neq \lambda, w_{4}: w_{3} w_{4}=w_{2} \wedge w_{1} w_{3} \in L\left(\mathrm{P}_{1}\right) \wedge w_{4} \in L\left(\mathrm{P}_{2}\right)\right) \\
\Leftrightarrow & \exists w_{1} \in \operatorname{lbreak}\left(C, L\left(\mathrm{P}_{1}\right), L\left(\mathrm{P}_{2}\right)\right): w_{1} \in \mathrm{P}_{1} \rightsquigarrow V_{1} \wedge V_{1}(n)=w \\
\Leftrightarrow & w \in \mathcal{T}^{\mathbb{P}}\left(n, \mathrm{P}_{1}, \operatorname{lbreak}\left(C, L\left(\mathrm{P}_{1}\right), L\left(\mathrm{P}_{2}\right)\right)\right)
\end{aligned}
$$

Equality (2) can be obtained in a similar way.

\section{MATCHING UNDER THE FIRST AND LONGEST MATCH POLICY}

In this section, we formally define the matching process on strings under the first and longest match disambiguation policy, and show that it guarantees a unique matching strategy. We also discuss the difference between the first and longest match policy and the XDuce policy.

Recall from Section 2.1 that the first and longest match policy consists of two disambiguation rules. The first match rule disambiguates a disjunction $\mathrm{P}_{1}+\mathrm{P}_{2}$ by giving higher priority to the first alternative $\mathrm{P}_{1}$. Moreover, disjunction distributes over concatenation. That is, when matching $w$ against $\left(\mathrm{P}_{1}+\mathrm{P}_{2}\right) \cdot \mathrm{P}_{3}, w$ should be first matched against $\mathrm{P}_{1} \cdot \mathrm{P}_{3}$ and it should only be matched against $\mathrm{P}_{2} \cdot \mathrm{P}_{3}$ when this fails. The longest match rule disambiguates the Kleene closure in patterns of the form $\mathrm{P}_{1}^{*} \cdot \mathrm{P}_{2}$ by requiring that $\mathrm{P}_{1}^{*}$ matches as much of the input as possible, still allowing the rest of the pattern to match. 
Example 6.1. Consider the matching of $a b$ against the pattern $(a+a \cdot b) \cdot(b+\varepsilon)$ of Figure 2(a). Then the whole pattern matches $a b$. Since disjunction distributes over concatenation, the first match rule requires us to first try to match $a b$ against $a \cdot(b+\varepsilon)$. This obviously succeeds. Since $a$ is matched by $a$ and $b$ by $(b+\varepsilon)$ in $a \cdot(b+\varepsilon)$, we associate $(a+a \cdot b)$ with $a$ and $(b+\varepsilon)$ with $b$ in $(a+a \cdot b) \cdot(b+\varepsilon)$.

In contrast, $(a \cdot a+b)$ is associated with $a b$ and $(b+\varepsilon)$ with $\varepsilon$ under the POSIX disambiguation policy, as we have shown in Example 4.1. Thus, under the first and longest match policy we no longer require that $\mathrm{P}_{1}$ matches as much as possible in a concatenation $\mathrm{P}_{1} \cdot \mathrm{P}_{2}$, unless $\mathrm{P}_{1}$ is a Kleene closure.

The matching relation $w \in \mathrm{P} \rightsquigarrow V$ under the first and longest match policy is formally defined in Figure 4. Rules Empty, Lab, KleEne, Or1, and Or2 are the same as in Figure 3. The difference with the POSIX policy lies in the treatment of concatenation patterns $\mathrm{P}_{1} \cdot \mathrm{P}_{2}$, for which we use the auxiliary relation $\left(w_{1}, w_{2}\right) \in$ $\mathrm{P}_{1} \cdot \mathrm{P}_{2} \rightsquigarrow\left(V_{1}, V_{2}\right)$. The intuitive meaning of this relation is that when matching $w_{1} w_{2}$ by $\mathbf{P}_{1} \cdot \mathrm{P}_{2}$ under the first and longest match policy, $\mathrm{P}_{1}$ will be responsible for matching prefix $w_{1}$ (with associations $V_{1}$ ), while $\mathrm{P}_{2}$ is responsible for matching suffix $w_{2}$ (with associations $V_{2}$ ). If $\mathrm{P}_{1}=\varepsilon$ or $\mathrm{P}_{1}=\sigma$, there is only one way to split the input word and no disambiguation is necessary, as expressed in rules CEMPTY and CLAB. Rules COR1 and COR2 express distribution of disjunction over concatenation, according to the first match rule. The longest match rule is expressed in CKLEENE. Note the resemblance of this rule with CONCAT of Figure 3. When matching $w$ against patterns of the form $\left(\mathrm{P}_{1} \cdot \mathrm{P}_{2}\right) \cdot \mathrm{P}_{3}$, we first determine the prefix $w_{1}$ that is matched by $\mathrm{P}_{1}$ by matching $w$ against $\mathrm{P}_{1} \cdot\left(\mathrm{P}_{2} \cdot \mathrm{P}_{3}\right)$. Then we determine which parts of the corresponding suffix are matched by $\mathrm{P}_{2}$ and $\mathrm{P}_{3}$ by matching this suffix against $P_{2} \cdot P_{3}$. The subword matched by $P_{1} \cdot P_{2}$ is then the concatenation of the subword matched by $\mathrm{P}_{1}$ and the subword matched by $\mathrm{P}_{2}$, as shown in rule CCON. Finally, rule CONCAT is used to convert from the auxiliary relation to the matching relation.

Example 6.2. As an example of the first match rule, consider the following matching derivation of $a b$ against pattern $(a+a \cdot b) \cdot(b+\varepsilon)$ :

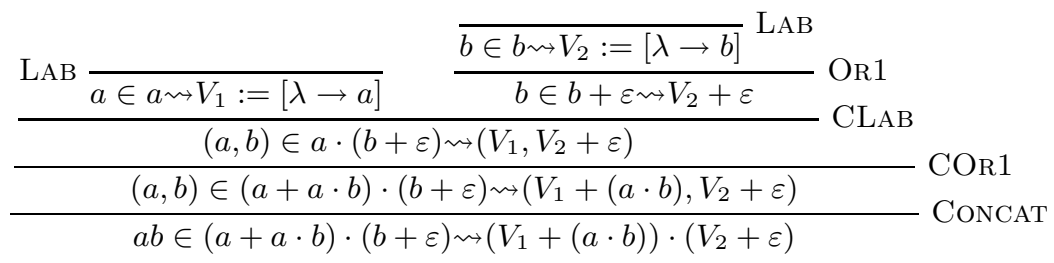

It is easily seen that the obtained association function $\left(V_{1}+(a \cdot b)\right) \cdot\left(V_{1}+\varepsilon\right)$ equals the association function $V^{\prime}$ from Example 3.1. For example,

$$
\left(\left(V_{1}+(a \cdot b)\right) \cdot\left(V_{2}+\varepsilon\right)\right)(1)=\left(V_{1}+(a \cdot b)\right)(\lambda)=V_{1}(\lambda)=a=V^{\prime}(1) .
$$

Likewise,

$$
\left(\left(V_{1}+(a \cdot b)\right) \cdot\left(V_{2}+\varepsilon\right)\right)(12)=\left(V_{1}+(a \cdot b)\right)(2)=\perp=V^{\prime}(12) .
$$




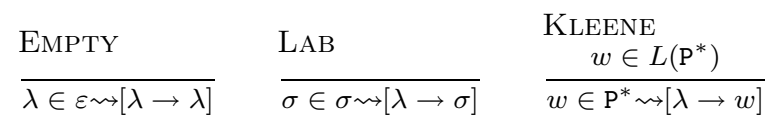

$$
\begin{aligned}
& \frac{\text { OR1 }}{w \in \mathrm{P}_{1} \rightsquigarrow V} \quad \begin{array}{l}
\text { OR2 } \\
w \in \mathrm{P}_{1}+\mathrm{P}_{2} \rightsquigarrow V+\mathrm{P}_{2}
\end{array} \quad \frac{w \quad w \notin L\left(\mathrm{P}_{1}\right)}{w \in \mathrm{P}_{1}+\mathrm{P}_{2} \rightsquigarrow \mathrm{P}_{1}+V} \\
& \text { CONCAT CEMPTY CLAB } \\
& \frac{\left(w_{1}, w_{2}\right) \in \mathrm{P}_{1} \cdot \mathrm{P}_{2} \rightsquigarrow\left(V_{1}, V_{2}\right)}{w_{1} w_{2} \in \mathrm{P}_{1} \cdot \mathrm{P}_{2} \rightsquigarrow V_{1} \cdot V_{2}} \quad \frac{w \in \mathrm{P} \rightsquigarrow V_{2}}{(\lambda, w) \in \varepsilon \cdot \mathrm{P} \rightsquigarrow\left([\lambda \rightarrow \lambda], V_{2}\right)} \quad \frac{\sigma \in \sigma \rightsquigarrow V_{1} \quad w \in \mathrm{P} \rightsquigarrow V_{2}}{(\sigma, w) \in \sigma \cdot \mathrm{P} \rightsquigarrow\left(V_{1}, V_{2}\right)} \\
& \text { COR1 } \\
& \left(w_{1}, w_{2}\right) \in \mathrm{P}_{1} \cdot \mathrm{P}_{3} \rightsquigarrow\left(V_{1}, V_{2}\right) \quad \frac{w_{1} w_{2} \notin L\left(\mathrm{P}_{1} \cdot \mathrm{P}_{3}\right)}{\left(w_{1}, w_{2}\right) \in\left(\mathrm{P}_{1}+\mathrm{P}_{2}\right) \cdot \mathrm{P}_{3} \rightsquigarrow\left(\mathrm{P}_{1}+1\right.} \\
& \overline{\left(w_{1}, w_{2}\right) \in\left(\mathrm{P}_{1}+\mathrm{P}_{2}\right) \cdot \mathrm{P}_{3} \rightsquigarrow\left(V_{1}+\mathrm{P}_{2}, V_{2}\right)} \quad \overline{\left(w_{1}, w_{2}\right) \in\left(\mathrm{P}_{1}+\mathrm{P}_{2}\right) \cdot \mathrm{P}_{3} \rightsquigarrow\left(\mathrm{P}_{1}+V_{1}, V_{2}\right)} \\
& \mathrm{CCON} \\
& \frac{\left(w_{1}, w_{2} w_{3}\right) \in \mathrm{P}_{1} \cdot\left(\mathrm{P}_{2} \cdot \mathrm{P}_{3}\right) \rightsquigarrow\left(V_{1}, W\right) \quad\left(w_{2}, w_{3}\right) \in \mathrm{P}_{2} \cdot \mathrm{P}_{3} \rightsquigarrow\left(V_{2}, V_{3}\right)}{\left(w_{1} w_{2}, w_{3}\right) \in\left(\mathrm{P}_{1} \cdot \mathrm{P}_{2}\right) \cdot \mathrm{P}_{3} \rightsquigarrow\left(V_{1} \cdot V_{2}, V_{3}\right)} \\
& \text { CKLeene } \\
& \frac{\neg\left(\exists w_{3} \neq \lambda, w_{4}: w_{3} w_{4}=\mathrm{P}_{1}^{*} \rightsquigarrow V_{1} \wedge w_{2} \in \mathrm{P}_{1} \rightsquigarrow w_{3} \in L\left(\mathrm{P}_{1}^{*}\right) \wedge w_{4} \in L\left(\mathrm{P}_{2}\right)\right)}{\left(w_{1}, w_{2}\right) \in \mathrm{P}_{1}^{*} \cdot \mathrm{P}_{2} \rightsquigarrow\left(V_{1}, V_{2}\right)}
\end{aligned}
$$

Fig. 4. The matching relation $w \in \mathrm{P} \rightsquigarrow V$ under the first and longest match disambiguation policy.

Example 6.3. As an example of the longest match rule, consider the following matching derivation of $a b$ against the pattern $(a+a \cdot b)^{*} \cdot(b+\varepsilon)$ of Figure $2(\mathrm{~b})$ :

$$
\begin{aligned}
& \text { EMPTY }
\end{aligned}
$$

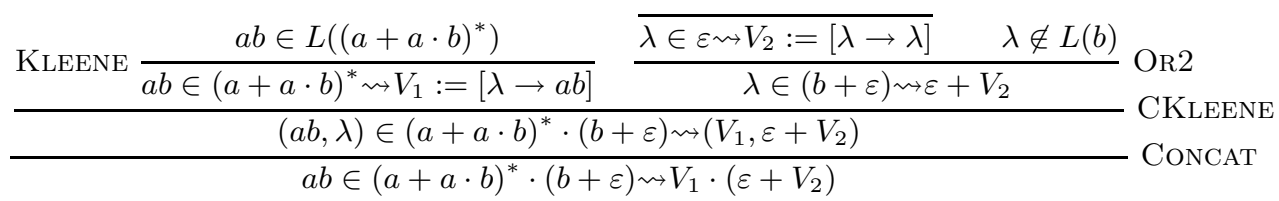

Here, $a b$ itself is matched by $(a+a \cdot b)^{*}$, while $(b+\varepsilon)$ matches $\lambda$ :

$$
\begin{aligned}
& \left(V_{1} \cdot\left(\varepsilon+V_{2}\right)\right)(1)=V_{1}(\lambda)=a b, \\
& \left(V_{1} \cdot\left(\varepsilon+V_{2}\right)\right)(2)=V_{2}(\lambda)=\lambda .
\end{aligned}
$$

Matching $a$ by $(a+a \cdot b)^{*}$ and $b$ by $(b+\varepsilon)$ will not work. Indeed, although it is possible to derive $a \in(a+a \cdot b)^{*} \rightsquigarrow W_{1}$ and $b \in(b+\varepsilon) \rightsquigarrow W_{2}$ for some associations $W_{1}$ and $W_{2}$, the third premise of CKLEENE will disable us to derive $(a, b) \in(a+a \cdot b)^{*} \cdot(b+\varepsilon) \rightsquigarrow\left(W_{1}, W_{2}\right)$.

As an analogy to Theorem 4.2 we have:

THEOREM 6.4. The matching relation of Figure 4 is well defined: 
(1) The matching relation is semantically correct: $w \in P \rightsquigarrow V$ iff $w \in L(P)$, and,

(2) The matching relation is unique: if $w \in P \rightsquigarrow V$ and $w \in P \rightsquigarrow W$ then $V=W$.

Proof. (1). The "only if" direction can be obtained by a straightforward induction on the matching derivation of $w \in \mathrm{P} \rightsquigarrow V$, with a case analysis on the last rule used. We highlight the case where this last rule is CONCAT. In that case $\mathbf{P}=\mathrm{P}_{1} \cdot \mathrm{P}_{2}$ and we can split up $w$ into $w_{1}$ and $w_{2}$ such that $\left(w_{1}, w_{2}\right) \in$ $\mathrm{P}_{1} \cdot \mathrm{P}_{2} \rightsquigarrow\left(V_{1}, V_{2}\right)$ with $V=V_{1} \cdot V_{2}$. A straightforward induction on the derivation of $\left(w_{1}, w_{2}\right) \in \mathrm{P}_{1} \cdot \mathrm{P}_{2} \rightsquigarrow\left(V_{1}, V_{2}\right)$ shows that $w_{1} \in L\left(\mathrm{P}_{1}\right)$ and $w_{2} \in L\left(\mathrm{P}_{2}\right)$. Hence, $w_{1} w_{2} \in L(\mathrm{P})$, as desired.

The "if" direction can be obtained by well-founded induction [Baader and Nipkow 1998] on $\mathrm{P}$ according to the well-founded relation $\succ$. Here, $\succ$ relates a pattern $\mathrm{P}$ with immediate subpatterns if $\mathrm{P} \neq\left(\mathrm{P}_{1} \cdot \mathrm{P}_{2}\right) \cdot \mathrm{P}_{3}$ and $\mathrm{P} \neq\left(\mathrm{P}_{1}+\mathrm{P}_{2}\right) \cdot \mathrm{P}_{3}$. It relates $\left(\mathrm{P}_{1} \cdot \mathrm{P}_{2}\right) \cdot \mathrm{P}_{3}$ with $\mathrm{P}_{2} \cdot \mathrm{P}_{3}$ and with $\mathrm{P}_{1} \cdot\left(\mathrm{P}_{2} \cdot \mathrm{P}_{3}\right)$ and it relates $\left(\mathrm{P}_{1}+\mathrm{P}_{2}\right) \cdot \mathrm{P}_{3}$ with $\mathrm{P}_{1} \cdot \mathrm{P}_{3}$ and with $\mathrm{P}_{2} \cdot \mathrm{P}_{3}$. The monotone embedding $\phi$ into the lexicographically ordered set $\mathbb{N} \times \mathbb{N}$ where $\phi(\mathrm{P})=(|\mathrm{P}|, 0)$ if $\mathrm{P} \neq \mathrm{P}_{1} \cdot \mathrm{P}_{2}$ and $\phi\left(\mathrm{P}_{1} \cdot \mathrm{P}_{2}\right)=\left(\left|\mathrm{P}_{1} \cdot \mathrm{P}_{2}\right|,\left|\mathrm{P}_{1}\right|\right)$ otherwise, shows that $\succ$ is well-founded [Baader and Nipkow 1998].

(2). By a straightforward induction on the matching derivation of $w \in \mathrm{P} \rightsquigarrow V$, with a case analysis on the last rule used. We highlight the case where this last rule is ConcAt. In that case, $\mathrm{P}=\mathrm{P}_{1} \cdot \mathrm{P}_{2}$ and we can split up $w$ into $w_{1}$ and $w_{2}$ such that $\left(w_{1}, w_{2}\right) \in \mathrm{P}_{1} \cdot \mathrm{P}_{2} \rightsquigarrow\left(V_{1}, V_{2}\right)$ with $V=V_{1} \cdot V_{2}$. Furthermore, since we also have $w \in \mathrm{P} \rightsquigarrow W$, we can also split up $w$ into $w_{3}$ and $w_{4}$ such that $\left(w_{3}, w_{4}\right) \in$ $\mathrm{P}_{1} \cdot \mathrm{P}_{2} \rightsquigarrow\left(W_{1}, W_{2}\right)$ with $W=W_{1} \cdot W_{2}$. A straightforward induction on the derivation of $\left(w_{1}, w_{2}\right) \in \mathrm{P}_{1} \cdot \mathrm{P}_{2} \rightsquigarrow\left(V_{1}, V_{2}\right)$ then shows that $w_{1}=w_{3}, w_{2}=w_{4}, V_{1}=W_{1}$, and $V_{2}=W_{2}$. Hence, $\in L\left(\mathrm{P}_{1}\right)$ and $w_{2} \in L\left(\mathrm{P}_{2}\right)$. Hence, $V=V_{1} \cdot V_{2}=W_{1} \cdot W_{2}=W$, as desired.

\subsection{Relation with the XDuce policy}

The disambiguation policy employed in XDuce [Hosoya 2000; Hosoya and Pierce 2002], $\mathbb{C D u c e}$ [Frisch et al. 2003], $\lambda^{\text {re }}$ [Tabuchi et al. 2002], and Perl [Wall et al. 2000] consists of two rules: first match and greedy match. The first match rule is the same as in the first and longest match policy. The greedy match rule disambiguates a Kleene closure and is defined in terms of the first match policy and recursion. Formally, the matching relation under the XDuce policy is obtained from the matching relation of the first and longest match policy by replacing rule CKLEENE as follows [Tabuchi et al. 2002]:

$$
\begin{aligned}
& \text { CKLEENE }^{\prime} \\
& \frac{\left(w_{1}, w_{2}\right) \in\left(\left(\mathrm{P}_{1} \cdot \mathrm{P}_{1}^{*}\right)+\varepsilon\right) \cdot \mathrm{P}_{2} \rightsquigarrow\left(V_{1}, V_{2}\right)}{\left(w_{1}, w_{2}\right) \in \mathrm{P}_{1}^{*} \cdot \mathrm{P}_{2} \rightsquigarrow\left(\left[\lambda \rightarrow V_{1}(\lambda)\right], V_{2}\right)}
\end{aligned}
$$

Here, it is assumed without loss of generality that $\lambda \notin L\left(\mathrm{P}_{1}\right)$.

The behavior of the greedy match rule was informally explained in [Hosoya 2000; Hosoya and Pierce 2002] as being the longest match rule. The intuition behind this was that, when trying to derive $w \in \mathrm{P}_{1}^{*} \cdot \mathrm{P}_{2} \rightsquigarrow V$, we will be forced by the first match rule to consider $\left(\mathrm{P}_{1} \cdot \mathrm{P}_{1}^{*}\right) \cdot \mathrm{P}_{2}$ before $\varepsilon \cdot \mathrm{P}_{2}$ at every expansion of $\mathrm{P}_{1}^{*} \cdot \mathrm{P}_{2}$. Since $\lambda \notin L\left(\mathrm{P}_{1}\right)$, this should require us to split $w$ into $w_{1} \in L\left(\mathrm{P}_{1}^{*}\right)$ and $w_{2} \in L\left(\mathrm{P}_{2}\right)$ such that $w_{2}$ is the smallest suffix of $w$ still matched by $\mathrm{P}_{2}$. This is, however, a false intuition. Indeed, 
the actual types to be $\{a b\}$ and $\{\lambda\}$ respectively. The main result of this paper can be stated as follows (to be proven later):

THEOREM 7.1. If $C$ is a regular language then $\mathcal{T}^{\mathbb{E L}}(m, P, C)$ is also regular, and can be effectively computed.

\subsection{The algorithm}

Algorithm 2 describes the type inference algorithm. As in Section 5.1, we will first explain the algorithm by informal reasoning, and prove its correctness later.

We observe that the type of the root node $\lambda$ is exactly the set of words in $C$ that can be matched by P. Indeed, if $w$ is successfully matched by $\mathrm{P}$ then $\lambda$ is associated to $w$ itself. If we need to calculate the type for a node other than $\lambda$, then $\mathrm{P}$ must be of the form $\mathrm{P}_{1}+\mathrm{P}_{2}$ or $\mathrm{P}_{1} \cdot \mathrm{P}_{2}$, since $\lambda$ is the only bindable node for the other patterns.

If $\mathrm{P}=\mathrm{P}_{1}+\mathrm{P}_{2}$ then we can make the same observations as in Section 5.1. Hence, $\mathcal{T}^{\mathbb{L L}}\left(1 n, \mathrm{P}_{1}+\mathrm{P}_{2}, C\right)$ equals $\mathcal{T}^{\mathbb{F L}}\left(n, \mathrm{P}_{1}, C\right)$ and $\mathcal{T}^{\mathbb{E L}}\left(2 n, \mathrm{P}_{1}+\mathrm{P}_{2}, C\right)$ equals $\mathcal{T}^{\mathbb{E L}}\left(n, \mathrm{P}_{2}, C-L\left(\mathrm{P}_{1}\right)\right)$.

If $\mathrm{P}=\mathrm{P}_{1} \cdot \mathrm{P}_{2}$ then we need to make a further case analysis:

- If $\mathrm{P}_{1}=\varepsilon$ or $\mathrm{P}_{1}=\sigma$, then $\mathrm{P}_{1}$ can only be associated with those words $w_{1}$ matched by $\mathrm{P}_{1}$ for which there exists some word $w_{2}$ matched by $\mathrm{P}_{2}$ such that $w_{1} w_{2} \in C$. Hence, $w_{1} \in C / L\left(\mathrm{P}_{2}\right)$ and $\mathcal{T}^{\mathbb{F L}}(1, \mathrm{P}, C)$ equals $\mathcal{T}^{\mathbb{R L}}\left(\lambda, \mathrm{P}_{1}, C / L\left(\mathrm{P}_{2}\right)\right)$. Likewise, subpatterns of $\mathrm{P}_{2}$ can only be associated to those subwords of a word $w_{2}$ matched by $\mathrm{P}_{2}$ for which there exists a $w_{1}$ matched by $\mathrm{P}_{1}$ such that $w_{1} w_{2} \in C$. Hence, we can calculate $\mathcal{T}^{\mathbb{E L}}(2 n, \mathrm{P}, C)$ by calculating $\mathcal{T}^{\mathbb{F L}}\left(n, \mathrm{P}_{2}, L\left(\mathrm{P}_{1}\right) \backslash C\right)$.

- For $\mathrm{P}=\mathrm{P}_{1}^{*} \cdot \mathrm{P}_{2}$, we again note the similarity between the POSIX and the first and longest match disambiguation policies. That is, $\mathrm{P}_{1}^{*}$ can only be associated to words $w_{1}$ matched by $\mathrm{P}_{1}^{*}$ for which there exists some $w_{2}$ matched by $\mathrm{P}_{2}$ such that $w_{1} w_{2} \in C$ and such that $w_{1}$ really is the longest possible prefix of $w_{1} w_{2}$ that can be matched by $\mathrm{P}_{1}^{*}$, still allowing the corresponding suffix to be matched by $\mathrm{P}_{2}$. Hence, $\mathcal{T}^{\mathbb{F L}}(1, \mathrm{P}, C)$ equals lbreak $\left(C, L\left(\mathrm{P}_{1}^{*}\right), L\left(\mathrm{P}_{2}\right)\right)$. Similarly, $\mathcal{T}^{\mathbb{F L}}(2 n, \mathrm{P}, C)$ equals $\mathcal{T}^{\mathbb{F L}}\left(n, \mathrm{P}_{2}, \operatorname{rbreak}\left(C, L\left(\mathrm{P}_{1}^{*}\right), L\left(\mathrm{P}_{2}\right)\right)\right)$.

- If $\mathrm{P}=\left(\mathrm{P}_{1}+\mathrm{P}_{2}\right) \cdot \mathrm{P}_{3}$, then a word can only be associated to a subpattern of $\mathrm{P}_{1}$ if it can be associated with $\mathrm{P}_{1}$ in $\mathrm{P}_{1} \cdot \mathrm{P}_{3}$. Hence, $\mathcal{T}^{\mathbb{L L}}(11 n, \mathrm{P}, C)$ equals $\mathcal{T}^{\mathbb{R L}}\left(1 n, \mathrm{P}_{1} \cdot \mathrm{P}_{3}, C\right)$. Likewise, a word can only be associated with $\mathrm{P}_{2}$ in $\mathrm{P}$ if it can be associated with $\mathrm{P}_{2}$ in $\mathrm{P}_{2} \cdot \mathrm{P}_{3}$ under context $C-L\left(\mathrm{P}_{1} \cdot \mathrm{P}_{3}\right)$. Hence, $\mathcal{T}^{\mathbb{F L}}(21 n, \mathrm{P}, C)$ equals

$$
\mathcal{T}^{\mathbb{F L}}\left(1 n, \mathrm{P}_{2} \cdot \mathrm{P}_{3}, C-L\left(\mathrm{P}_{1} \cdot \mathrm{P}_{3}\right)\right) .
$$

Finding the words that can be bound to $\left(\mathrm{P}_{1}+\mathrm{P}_{2}\right)$ resolves to calculating the union of words that can be bound to $\mathrm{P}_{1}$ or $\mathrm{P}_{2}$. Words can be bound to subpatterns of $\mathrm{P}_{3}$ if they are subwords of a word $w_{3}$ matched by $\mathrm{P}_{3}$ for which there either exits a word $w_{1}$ matched by $\mathrm{P}_{1}$ such that $w_{1} w_{3} \in C$, or a word $w_{2}$ matched by $\mathrm{P}_{2}$ such that $w_{2} w_{3} \in C$ but $w_{2} w_{3} \notin L\left(\mathrm{P}_{1} \cdot \mathrm{P}_{3}\right)$. Hence, $\mathcal{T}^{\mathbb{R}}(2 n, \mathrm{P}, C)$ equals

$$
\mathcal{T}^{\mathbb{E L}}\left(2 n, \mathrm{P}_{1} \cdot \mathrm{P}_{3}, C\right) \cup \mathcal{T}^{\mathbb{F L}}\left(2 n, \mathrm{P}_{2} \cdot \mathrm{P}_{3}, C-L\left(\mathrm{P}_{1} \cdot \mathrm{P}_{3}\right)\right)
$$

- Calculating the types of subpatterns of $\mathrm{P}_{1}, \mathrm{P}_{2}$, or $\mathrm{P}_{3}$ in $\mathrm{P}=\left(\mathrm{P}_{1} \cdot \mathrm{P}_{2}\right) \cdot \mathrm{P}_{3}$ is simply a matter of calculating the type of the corresponding subpatterns in $\mathrm{P}^{\prime}=\mathrm{P}_{1} \cdot\left(\mathrm{P}_{2} \cdot \mathrm{P}_{3}\right)$. The type of $\left(\mathrm{P}_{1} \cdot \mathrm{P}_{2}\right)$ is a bit more difficult to find. By definition 


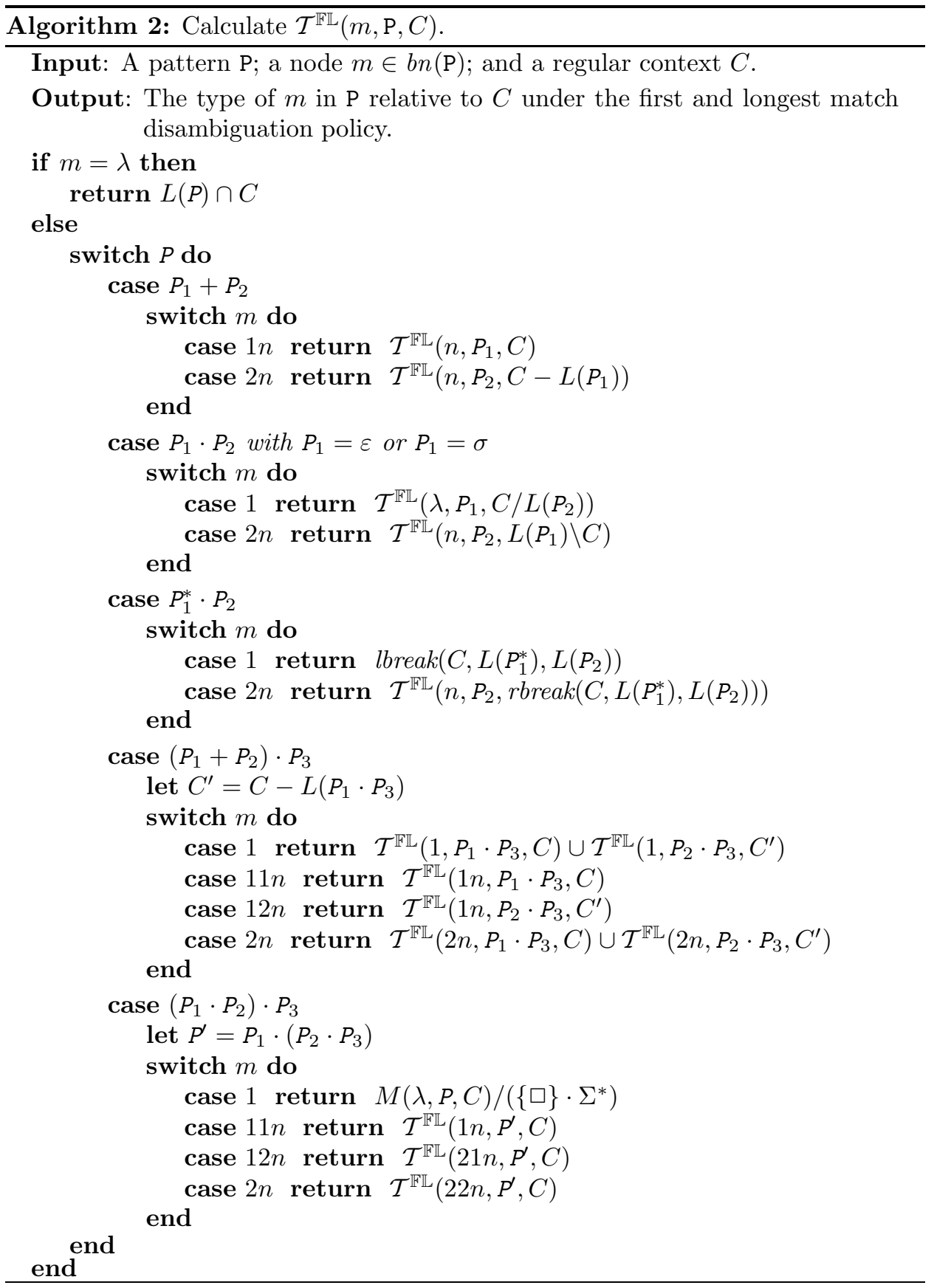


of the matching relation, $\left(\mathrm{P}_{1} \cdot \mathrm{P}_{2}\right)$ can only be associated to those words $w_{1} w_{2}$ for which there exists a $w_{3}$ such that $w_{1} w_{2} w_{3} \in C,\left(w_{1}, w_{2} w_{3}\right) \in \mathrm{P}^{\prime} \rightsquigarrow\left(V_{1}, W\right)$ and $\left(w_{2}, w_{3}\right) \in \mathrm{P}_{2} \cdot \mathrm{P}_{3} \rightsquigarrow\left(V_{2}, V_{3}\right)$. It is tempting to say that this means that the type of $\left(\mathrm{P}_{1} \cdot \mathrm{P}_{2}\right)$ is exactly the right quotient of $L(\mathrm{P}) \cap C$ by $L\left(\mathrm{P}_{3}\right)$. This is incorrect however. Indeed, consider the pattern $(a \cdot(a \cdot b+a)) \cdot(b+\varepsilon)$ and context $C=\{a a b, a a b b\}$. Then $\{a a b, a a b b\} /\{b, \lambda\}=\{a a, a a b, a a b b\}$, which is too big since $a a$ will never be associated with $(a \cdot(a \cdot b+a))$ under context $C$. Indeed, because of the first match policy, every word in $C$ will first be matched against $a \cdot(a \cdot b) \cdot(b+\varepsilon)$, which always succeeds. Hence, the type of $(a \cdot(a \cdot b+a))$ is $\{a a b, a a b b\}$. In order to correctly calculate the type of $\left(\mathrm{P}_{1} \cdot \mathrm{P}_{2}\right)$ in $\left(\mathrm{P}_{1} \cdot \mathrm{P}_{2}\right) \cdot \mathrm{P}_{3}$ we will use marked languages, which are defined as follows.

A marked language is a set of words of the form $w_{1} \square w_{2}$. The breaking of a context by two languages, as defined in Section 5.2, is an example of a marked language. Here, we will use the $\square$ marker to record that matching $w_{1} w_{2}$ against a concatenation $\mathrm{P}_{1} \cdot \mathrm{P}_{2}$ results in $w_{1}$ being matched by $\mathrm{P}_{1}$ and $w_{2}$ being matched by $\mathrm{P}_{2}$. We therefore define, for every pattern $\mathrm{P}$, the marked language $M(m, \mathrm{P}, C)$ of a node $m \in b n(\mathrm{P})$ with $\mathrm{P}(m)=$. under context $C$ as follows:

$$
M(m, \mathrm{P}, C)=\left\{w_{1} \square w_{2} \mid \exists w^{\prime} \in C, w^{\prime} \in \mathrm{P} \rightsquigarrow V, V(m 1)=w_{1}, V(m 2)=w_{2}\right\} .
$$

It is clear that, for $\mathrm{P}=\left(\mathrm{P}_{1} \cdot \mathrm{P}_{2}\right) \cdot \mathrm{P}_{3}, \mathcal{T}^{\mathbb{L}}(1, \mathrm{P}, C)=M(\lambda, \mathrm{P}, C) /\left(\{\square\} \cdot \Sigma^{*}\right)$. So, doing type inference for node 1 in $\mathrm{P}$ is simply a matter of calculating $M(\lambda, \mathrm{P}, C)$. We use Algorithm 3 for this purpose.

Algorithm 3 uses the following reasoning to compute $M\left(m,\left(\mathrm{P}_{1} \cdot \mathrm{P}_{2}\right) \cdot \mathrm{P}_{3}, C\right)$. Matching rule CCON states that if we want to know which part of word $w$ is matched by $\left(\mathrm{P}_{1} \cdot \mathrm{P}_{2}\right)$ when matching $w$ by $\mathrm{P}$, then we first determine how it is broken up against $\mathrm{P}^{\prime}=\mathrm{P}_{1} \cdot\left(\mathrm{P}_{2} \cdot \mathrm{P}_{3}\right)$. Suppose that $w=w_{1} v$, that $\mathrm{P}_{1}$ is responsible for matching $w_{1}$, and that $\left(\mathrm{P}_{2} \cdot \mathrm{P}_{3}\right)$ is responsible for matching $v$ when matching $w$ by $\mathrm{P}^{\prime}$. Next, we determine how $v$ is broken up by the matching against $\left(\mathrm{P}_{2} \cdot \mathrm{P}_{3}\right)$. Suppose that $v=w_{2} w_{3}$, that $\mathrm{P}_{2}$ is responsible for matching $w_{2}$, and that $\mathrm{P}_{3}$ is responsible for matching $w_{3}$. Then CCON states that $w_{1} w_{2}$ is matched by $\left(\mathrm{P}_{1} \cdot \mathrm{P}_{2}\right)$ in $\mathrm{P}$ and $w_{3}$ by $\mathrm{P}_{3}$ in $\mathrm{P}$. Note that by definition, $w_{1} \square w_{2} w_{3} \in M\left(\lambda, \mathrm{P}^{\prime}, C\right)$ and $w_{2} \square w_{3} \in M\left(2, \mathrm{P}^{\prime}, C\right)$. Hence, if we already have $M\left(\lambda, \mathrm{P}^{\prime}, C\right)$ and $M\left(2, \mathrm{P}^{\prime}, C\right)$, it suffices to "link" these two sets correctly together in order to calculate $M(\lambda, \mathrm{P}, C)$. We therefore define the redistribution of two marked languages $M_{1}$ and $M_{2}$, denoted by redistrib $\left(M_{1}, M_{2}\right)$, to be the marked language

$$
\text { redistrib }\left(M_{1}, M_{2}\right):=\left\{w_{1} w_{2} \square w_{3} \mid w_{1} \square w_{2} w_{3} \in M_{1}, w_{2} \square w_{3} \in M_{2}\right\} .
$$

By the reasoning made above, it is intuitively clear that

$$
M(\lambda, \mathrm{P}, C)=\text { redistrib }\left(M\left(\lambda, \mathrm{P}^{\prime}, C\right), M\left(2, \mathrm{P}^{\prime}, C\right)\right) .
$$

We will prove this claim formally in the following section. Of course, we need a way to actually calculate the redistribution:

LEMMA 7.2. If $M_{1}$ and $M_{2}$ are regular, then so is redistrib $\left(M_{1}, M_{2}\right)$, which can effectively be computed. 


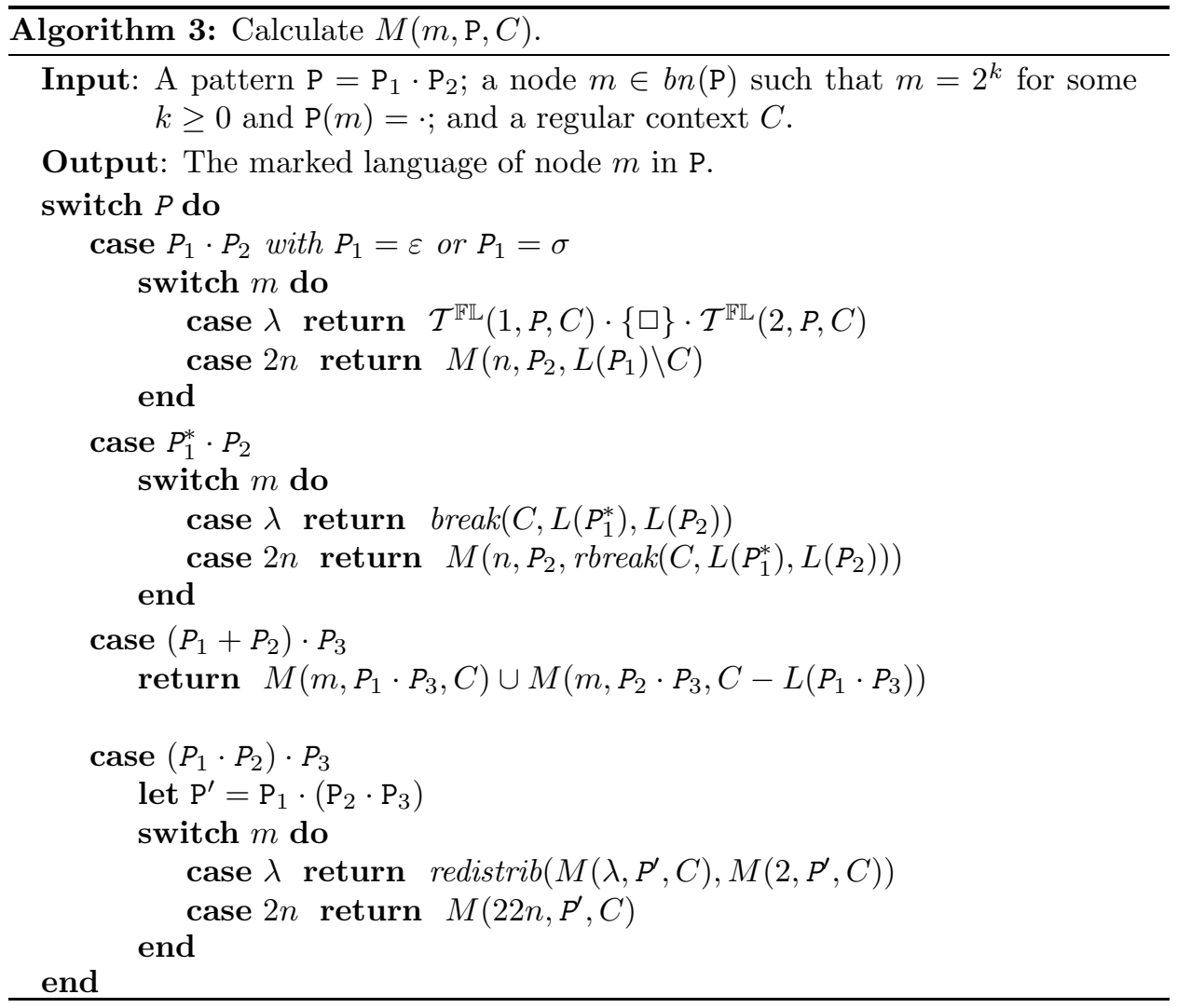

Proof. We introduce two operations on regular languages:

$$
\begin{aligned}
\iota(L) & =\left\{w_{1} \square w_{2} \square w_{3} \mid w_{1} \square w_{2} w_{3} \in L\right\}, \\
\pi_{1}(L) & =\left\{w_{1} w_{2} \square w_{3} \mid w_{1} \square w_{2} \square w_{3} \in L\right\} .
\end{aligned}
$$

It is clear that regular languages are closed under these two operations. For example, we can obtain an automaton for $\iota(L)$ by modifying an automaton for $L$ to allow the reading of a second $\square$ after the first, which is then ignored. The lemma then follows since redistrib $\left(M_{1}, M_{2}\right)=\pi_{1}\left(\iota\left(M_{1}\right) \cap\left(\Sigma^{*} \cdot\{\square\} \cdot M_{2}\right)\right)$.

Now we have a way to calculate the marked language $M\left(\lambda,\left(\mathrm{P}_{1} \cdot \mathrm{P}_{2}\right) \cdot \mathrm{P}_{3}, C\right)$ if we can calculate $M\left(\lambda, \mathrm{P}_{1} \cdot\left(\mathrm{P}_{2} \cdot \mathrm{P}_{3}\right), C\right)$ and $M\left(2, \mathrm{P}_{1} \cdot\left(\mathrm{P}_{2} \cdot \mathrm{P}_{3}\right), C\right)$. Algorithm 3 calculates these marked languages by case analysis on $\mathrm{P}_{1} \cdot\left(\mathrm{P}_{2} \cdot \mathrm{P}_{3}\right)$, recursively calling itself when necessary. To do so, we only have to be able to calculate $M\left(m, \mathrm{P}^{\prime \prime}, C\right)$ for patterns $\mathrm{P}^{\prime \prime}$ of the form $\mathrm{P}_{1}^{\prime \prime} \cdot \mathrm{P}_{2}^{\prime \prime}$ and nodes $m=2^{k} \in b n\left(\mathrm{P}^{\prime \prime}\right)$ with $\mathrm{P}^{\prime \prime}(m)=\cdot$. That is, Algorithm 3 only needs to recursively call itself on such arguments.

Getting an understanding of this algorithm largely involves the same reasoning as for $\mathcal{T}^{\mathbb{F L}}\left(n, \mathrm{P}^{\prime \prime}, C\right)$. For instance, suppose $\mathrm{P}^{\prime \prime}=\mathrm{P}_{1}^{\prime \prime} \cdot \mathrm{P}_{2}^{\prime \prime}$ with $\mathrm{P}_{1}^{\prime \prime}=\varepsilon$ or $\mathrm{P}_{1}^{\prime \prime}=\sigma$. If $w \in C$ is matched by $\mathrm{P}^{\prime \prime}$, then $w$ must be able to be split in words $w_{1}$ matched by $\mathrm{P}_{1}^{\prime \prime}$ and $w_{2}$ matched by $\mathrm{P}_{2}^{\prime \prime}$. Since $L\left(\mathrm{P}_{1}^{\prime \prime}\right)$ contains only one word, there can be no ambiguity in determining $w_{1}$ and $w_{2}$. Hence, $M\left(\lambda, \mathrm{P}^{\prime \prime}, C\right)$ equals $\mathcal{T}^{\mathbb{R}}\left(1, \mathrm{P}^{\prime \prime}, C\right)$. $\{\square\} \cdot \mathcal{T}^{\mathbb{E L}}\left(2, \mathrm{P}^{\prime \prime}, C\right)$. Likewise, $M\left(2 n, \mathrm{P}^{\prime \prime}, C\right)$ equals $M\left(n, \mathrm{P}_{2}^{\prime \prime}, L\left(\mathrm{P}_{1}^{\prime \prime}\right) \backslash C\right)$. For the other 
cases, similar reasonings can be done and we will therefore not elaborate further on the working of Algorithm 3 here. Its correctness will be formally demonstrated in the next section.

\subsection{Proof of Correctness}

It is not immediately clear that Algorithms 2 and 3 terminate on every input. We will first prove they do:

Proposition 7.3. Algorithms 2 and 3 terminate on every input.

Proof. A relation $\succ$ on a set $A$ well-founded (or terminating) if there is no infinite decreasing sequence $a_{1} \succ a_{2} \succ a_{3} \succ \ldots$ [Baader and Nipkow 1998]. We will define a well-founded binary relation $\sqsupset$ on the set of all patterns. Termination of both algorithms then follows as they only recursively call themselves with "smaller" inputs (according to $\sqsupset$ ).

We define $\sqsupset$ to relate a pattern $\mathrm{P}$ with its immediate subpatterns if $\mathrm{P} \neq\left(\mathrm{P}_{1} \cdot \mathrm{P}_{2}\right) \cdot \mathrm{P}_{3}$ and $\mathrm{P} \neq\left(\mathrm{P}_{1}+\mathrm{P}_{2}\right) \cdot \mathrm{P}_{3}$. It relates $\left(\mathrm{P}_{1} \cdot \mathrm{P}_{2}\right) \cdot \mathrm{P}_{3}$ with $\mathrm{P}_{2} \cdot \mathrm{P}_{3}$ and with $\mathrm{P}_{1} \cdot\left(\mathrm{P}_{2} \cdot \mathrm{P}_{3}\right)$. It relates $\left(\mathrm{P}_{1}+\mathrm{P}_{2}\right) \cdot \mathrm{P}_{3}$ with $\mathrm{P}_{1} \cdot \mathrm{P}_{3}$ and with $\mathrm{P}_{2} \cdot \mathrm{P}_{3}$. The monotone embedding $\phi$ into the lexicographically ordered $\mathbb{N} \times \mathbb{N}$ where $\phi(\mathrm{P})=(|\mathrm{P}|, 0)$ if $\mathrm{P} \neq \mathrm{P}_{1} \cdot \mathrm{P}_{2}$ and $\left.\phi\left(\mathrm{P}_{1} \cdot \mathrm{P}_{2}\right)=\left(\left|\mathrm{P}_{1} \cdot \mathrm{P}_{2}\right|\right),\left|\mathrm{P}_{1}\right|\right)$ otherwise, shows that $\sqsupset$ is well-founded [Baader and Nipkow 1998].

Let $(m, \mathrm{P}, C)$ be a valid input of Algorithm 3. It is clear that Algorithm 3 directly calls itself only on inputs $\left(m^{\prime}, \mathrm{P}^{\prime}, C^{\prime}\right)$ with $\mathrm{P} \sqsupset \mathrm{P}^{\prime}$. If $\mathrm{P}=\mathrm{P}_{1} \cdot \mathrm{P}_{2}$ and $m=\lambda$ with $\mathrm{P}_{1}=\varepsilon$ or $\mathrm{P}_{1}=\sigma$ then Algorithm 3 calls Algorithm 2 with arguments $(1, \mathrm{P}, C)$ and $(2, \mathrm{P}, C)$. On these arguments, Algorithm 2 will call itself with arguments $\left(\lambda, \mathrm{P}_{1}, C / L\left(\mathrm{P}_{2}\right)\right)$ and $\left(\lambda, \mathrm{P}_{2}, L\left(\mathrm{P}_{1}\right) \backslash C\right)$. On these recursive calls, Algorithm 2 terminates in one step. Hence, Algorithm 3 terminates on every input.

Let $(m, \mathrm{P}, C)$ be the input of Algorithm 2. It is clear that Algorithm 2 directly calls itself only on inputs $\left(m^{\prime}, \mathrm{P}^{\prime}, C^{\prime}\right)$ where $\mathrm{P} \sqsupset \mathrm{P}^{\prime}$. If $\mathrm{P}=\left(\mathrm{P}_{1} \cdot \mathrm{P}_{2}\right) \cdot \mathrm{P}_{3}$ and $m=1$, Algorithm 3 is called, which always terminates. Hence, Algorithm 2 terminates on every input.

We will now formally prove the correctness of Algorithms 2 and 3, thereby also proving Theorem 7.1.

LEMmA 7.4. If $w \in P \rightsquigarrow V$ then $V(\lambda)=w$, and if $\left(w_{1}, w_{2}\right) \in P_{1} \cdot P_{2} \rightsquigarrow\left(V_{1}, V_{2}\right)$ then $V_{1}(\lambda)=w_{1}$ and $V_{2}(\lambda)=w_{2}$.

Proof. By a straightforward induction on the matching derivation.

Proposition 7.5. $\mathcal{T}^{\mathbb{E L}}(\lambda, P, C)=L(P) \cap C$ for any pattern $P$.

Proof. Similar to the proof of Proposition 5.4.

Proposition 7.6. For $P=P_{1}+P_{2}$, the following equalities hold:

(1) $\mathcal{T}^{\mathbb{R L}}(1 n, P, C)=\mathcal{T}^{\mathbb{R L}}\left(n, P_{1}, C\right)$

(2) $\mathcal{T}^{\mathbb{L}}(2 n, P, C)=\mathcal{T}^{\mathbb{F L}}\left(n, P_{2}, C-L\left(P_{1}\right)\right)$

Proof. Similar to the proof of Proposition 5.5.

Proposition 7.7. If $P=P_{1} \cdot P_{2}$ with $P_{1}=\varepsilon$ or $P_{1}=\sigma$, then the following equalities hold: 
(1) $\mathcal{T}^{\mathbb{F L}}(1, P, C)=\mathcal{T}^{\mathbb{F L}}\left(\lambda, P_{1}, C / L\left(P_{2}\right)\right)$

(2) $\mathcal{T}^{\mathbb{F L}}(2 n, P, C)=\mathcal{T}^{\mathbb{F L}}\left(n, P_{2},\left(L\left(P_{1}\right) \backslash C\right)\right)$

(3) $M(\lambda, P, C)=\mathcal{T}^{\mathbb{F L}}(1, P, C) \cdot\{\square\} \cdot \mathcal{T}^{\mathbb{F L}}(2, P, C)$

(4) $M(2 n, P, C)=M\left(n, P_{2}, L\left(P_{1}\right) \backslash C\right)$

Proof. We prove the case where $\mathrm{P}_{1}=\sigma$, the case where $\mathrm{P}_{1}=\varepsilon$ is similar. Note that if $\mathrm{P}_{1}=\sigma$ the top of any matching derivation of $w^{\prime} \in \mathrm{P} \rightsquigarrow V$ has the following form:

$$
\frac{\frac{\cdots}{w_{1} \in \sigma \rightsquigarrow V_{1}} \quad \frac{\cdots}{w_{2} \in \mathrm{P}_{2} \rightsquigarrow V_{2}}}{\frac{\left(w_{1}, w_{2}\right) \in \mathrm{P} \rightsquigarrow\left(V_{1}, V_{2}\right)}{w^{\prime}=w_{1} w_{2} \in \mathrm{P} \rightsquigarrow V=V_{1} \cdot V_{2}}} \text { CLAB }
$$

Equality (1) then readily follows by Theorem 6.4 :

$$
\begin{aligned}
w \in \mathcal{T}^{\mathbb{F L}}(1, \mathrm{P}, C) & \Leftrightarrow \exists w^{\prime} \in C: w^{\prime} \in \mathrm{P} \rightsquigarrow V \wedge V(1)=w \\
& \Leftrightarrow \exists w_{1}, w_{2}: w_{1} w_{2} \in C \wedge w_{1} \in \sigma \rightsquigarrow V_{1} \wedge w_{2} \in \mathrm{P}_{2} \rightsquigarrow V_{2} \wedge V_{1}(\lambda)=w \\
& \Leftrightarrow \exists w_{1}, w_{2}: w_{1} w_{2} \in C \wedge w_{1} \in \sigma \rightsquigarrow V_{1} \wedge w_{2} \in L\left(\mathrm{P}_{2}\right) \wedge V_{1}(\lambda)=w \\
& \Leftrightarrow \exists w_{1} \in C / L\left(\mathrm{P}_{2}\right): w_{1} \in \sigma \rightsquigarrow V_{1} \wedge V_{1}(\lambda)=w \\
& \Leftrightarrow w \in \mathcal{T}^{\mathbb{F L}}\left(\lambda, \sigma, C / L\left(\mathrm{P}_{2}\right)\right)
\end{aligned}
$$

Equalities (2) and (4) can be proven similarly. Equality (3) readily follows by Theorem 6.4 and equalities (1) and (2):

$$
\begin{aligned}
v_{1} \square v_{2} \in M(\lambda, \mathrm{P}, C) \Leftrightarrow & \exists w^{\prime} \in C: w^{\prime} \in \mathrm{P} \rightsquigarrow V \wedge V(1)=v_{1} \wedge V(2)=w_{2} \\
\Leftrightarrow & \exists w_{1}, w_{2}: w_{1} w_{2} \in C \wedge w_{1} \in \sigma \rightsquigarrow V_{1} \wedge w_{2} \in \mathrm{P}_{2} \rightsquigarrow V_{2} \\
& \wedge V_{1}(\lambda)=v_{1} \wedge V_{2}(\lambda)=v_{2} \\
\Leftrightarrow & \exists w_{1}, w_{2}: w_{1} w_{2} \in C \wedge w_{1} \in L(\sigma) \wedge w_{1} \in \sigma \rightsquigarrow V_{1} \\
& \wedge w_{2} \in L\left(\mathrm{P}_{2}\right) \wedge w_{2} \in \mathrm{P}_{2} \rightsquigarrow V_{2} \wedge V_{1}(\lambda)=v_{1} \wedge V_{2}(\lambda)=v_{2} \\
\Leftrightarrow & \exists w_{1} \in C / L\left(\mathrm{P}_{2}\right), w_{2} \in L(\sigma) \backslash C: w_{1} \in \sigma \rightsquigarrow V_{1} \wedge w_{2} \in \mathrm{P}_{2} \rightsquigarrow V_{2} \\
& \wedge V_{1}(\lambda)=v_{1} \wedge V_{2}(\lambda)=v_{2} \\
\Leftrightarrow & v_{1} \square v_{2} \in \mathcal{T}^{\mathbb{F L}}\left(\lambda, \sigma, C / L\left(\mathrm{P}_{2}\right)\right) \cdot\{\square\} \cdot \mathcal{T}^{\mathbb{F L}}\left(\lambda, \mathrm{P}_{2}, L(\sigma) \backslash C\right) \\
\Leftrightarrow & v_{1} \square v_{2} \in \mathcal{T}^{\mathbb{F L}}(1, \mathrm{P}, C) \cdot\{\square\} \cdot \mathcal{T}^{\mathbb{F L}}(2, \mathrm{P}, C)
\end{aligned}
$$

Proposition 7.8. If $P=P_{1}^{*} \cdot P_{2}$, then the following equalities hold:

(1) $\mathcal{T}^{\mathbb{F L}}(1, P, C)=\operatorname{lbreak}\left(C, L\left(P_{1}^{*}\right), L\left(P_{2}\right)\right)$

(2) $\mathcal{T}^{\mathbb{F L}}(2 n, P, C)=\mathcal{T}^{\mathbb{F L}}\left(n, P_{2}, \operatorname{rbreak}\left(C, L\left(P_{1}^{*}\right), L\left(P_{2}\right)\right)\right)$

(3) $M(\lambda, P, C)=\operatorname{break}\left(C, L\left(P_{1}^{*}\right), L\left(P_{2}\right)\right)$

(4) $M(2 n, P, C)=M\left(n, P_{2}, \operatorname{rbreak}\left(C, L\left(P_{1}^{*}\right), L\left(P_{2}\right)\right)\right)$ 
Proof. Note that for any derivation of $w^{\prime} \in \mathrm{P} \rightsquigarrow V$, the top must look like:

$$
\begin{aligned}
& \frac{\cdots}{w_{1} \in \mathrm{P}_{1}^{*} \rightsquigarrow V_{1}} \quad \frac{\cdots}{w_{2} \in \mathrm{P}_{2} \rightsquigarrow V_{2}} \\
& \frac{\frac{\neg\left(\exists w_{3} \neq \lambda, w_{4}: w_{3} w_{4}=w_{2} \wedge w_{1} w_{3} \in L\left(\mathrm{P}_{1}^{*}\right) \wedge w_{4} \in L\left(\mathrm{P}_{2}\right)\right)}{\left(w_{1}, w_{2}\right) \in \mathrm{P} \rightsquigarrow\left(V_{1}, V_{2}\right)} \text { CKLEENE }}{w^{\prime}=w_{1} w_{2} \in \mathrm{P} \rightsquigarrow V=V_{1} \cdot V_{2}} \text { CONCAT }
\end{aligned}
$$

Also note that $V_{1}(\lambda)=w_{1}$ and $V_{2}(\lambda)=w_{2}$ by Lemma 7.4. From these observations and Theorem 6.4 equality (1) readily follows:

$$
\begin{aligned}
w \in & \mathcal{T}^{\mathbb{E L}}(1, \mathrm{P}, C) \\
\Leftrightarrow & \exists w^{\prime} \in C: w^{\prime} \in \mathrm{P} \rightsquigarrow V \wedge V(1)=w \\
\Leftrightarrow & \exists w_{1}, w_{2}: w_{1} w_{2} \in C \wedge w_{1} \in \mathrm{P}_{1}^{*} \rightsquigarrow V_{1} \wedge w_{2} \in \mathrm{P}_{2} \rightsquigarrow V_{2} \wedge V_{1}(\lambda)=w \\
& \wedge \neg\left(\exists w_{3} \neq \lambda, w_{4}: w_{3} w_{4}=w_{2} \wedge w_{1} w_{3} \in L\left(\mathrm{P}_{1}^{*}\right) \wedge w_{4} \in L\left(\mathrm{P}_{2}\right)\right) \\
\Leftrightarrow & \exists w_{2}: w w_{2} \in C \wedge w \in \mathrm{P}_{1}^{*} \rightsquigarrow V_{1} \wedge w_{2} \in \mathrm{P}_{2} \rightsquigarrow V_{2} \\
& \wedge \neg\left(\exists w_{3} \neq \lambda, w_{4}: w_{3} w_{4}=w_{2} \wedge w w_{3} \in L\left(\mathrm{P}_{1}^{*}\right) \wedge w_{4} \in L\left(\mathrm{P}_{2}\right)\right) \\
\Leftrightarrow & \exists w_{2}: w w_{2} \in C \wedge w \in L\left(\mathrm{P}_{1}^{*}\right) \wedge w_{2} \in L\left(\mathrm{P}_{2}\right) \\
& \wedge \neg\left(\exists w_{3} \neq \lambda, w_{4}: w_{3} w_{4}=w_{2} \wedge w w_{3} \in L\left(\mathrm{P}_{1}^{*}\right) \wedge w_{4} \in L\left(\mathrm{P}_{2}\right)\right) \\
\Leftrightarrow & w \in l b r e a k\left(C, L\left(\mathrm{P}_{1}^{*}\right), L\left(\mathrm{P}_{2}\right)\right)
\end{aligned}
$$

Equality (3) can be obtained by a similar reasoning:

$$
\begin{aligned}
v_{1} \square v_{2} \in & M(\lambda, \mathrm{P}, C) \\
\Leftrightarrow & \exists w^{\prime} \in C: w^{\prime} \in \mathrm{P} \rightsquigarrow V \wedge V(1)=v_{1} \wedge V(2)=v_{2} \\
\Leftrightarrow & \exists w_{1}, w_{2}: w_{1} w_{2} \in C \wedge w_{1} \in \mathrm{P}_{1}^{*} \rightsquigarrow V_{1} \wedge w_{2} \in \mathrm{P}_{2} \rightsquigarrow V_{2} \\
& \wedge V_{1}(\lambda)=v_{1} \wedge V_{2}(\lambda)=v_{2} \\
& \wedge \neg\left(\exists w_{3} \neq \lambda, w_{4}: w_{3} w_{4}=w_{2} \wedge w_{1} w_{3} \in L\left(\mathrm{P}_{1}^{*}\right) \wedge w_{4} \in L\left(\mathrm{P}_{2}\right)\right) \\
\Leftrightarrow & v_{1} v_{2} \in C \wedge v_{1} \in \mathrm{P}_{1}^{*} \rightsquigarrow V_{1} \wedge v_{2} \in \mathrm{P}_{2} \rightsquigarrow V_{2} \\
& \wedge \neg\left(\exists w_{3} \neq \lambda, w_{4}: w_{3} w_{4}=v_{2} \wedge v_{1} w_{3} \in L\left(\mathrm{P}_{1}^{*}\right) \wedge w_{4} \in L\left(\mathrm{P}_{2}\right)\right) \\
\Leftrightarrow & v_{1} v_{2} \in C \wedge v_{1} \in L\left(\mathrm{P}_{1}^{*}\right) \wedge v_{2} \in L\left(\mathrm{P}_{2}^{*}\right) \\
& \wedge \neg\left(\exists w_{3} \neq \lambda, w_{4}: w_{3} w_{4}=v_{2} \wedge v_{1} w_{3} \in L\left(\mathrm{P}_{1}^{*}\right) \wedge w_{4} \in L\left(\mathrm{P}_{2}\right)\right) \\
\Leftrightarrow & v_{1} \square v_{2} \in \operatorname{break}\left(C, L\left(\mathrm{P}_{1}^{*}\right), L\left(\mathrm{P}_{2}\right)\right)
\end{aligned}
$$

Equalities (2) and (4) can be proven similarly.

Proposition 7.9. If $P=\left(P_{1}+P_{2}\right) \cdot P_{3}, P_{1}^{\prime}=P_{1} \cdot P_{3}$, and $P_{2}^{\prime}=P_{2} \cdot P_{3}$, then the following equalities hold:

(1) $\mathcal{T}^{\mathbb{F L}}(1, P, C)=\mathcal{T}^{\mathbb{R L}}\left(1, P_{1}^{\prime}, C\right) \cup \mathcal{T}^{\mathbb{F L}}\left(1, P_{2}^{\prime}, C-L\left(P_{1}^{\prime}\right)\right)$

(2) $\mathcal{T}^{\mathbb{F L}}(11 n, P, C)=\mathcal{T}^{\mathbb{F L}}\left(1 n, P_{1}^{\prime}, C\right)$

(3) $\mathcal{T}^{\mathbb{F L}}(12 n, P, C)=\mathcal{T}^{\mathbb{F L}}\left(1 n, P_{2}^{\prime}, C-L\left(P_{1}^{\prime}\right)\right)$

(4) $\mathcal{T}^{\mathbb{E L}}(2 n, P, C)=\mathcal{T}^{\mathbb{F L}}\left(2 n, P_{1}^{\prime}, C\right) \cup \mathcal{T}^{\mathbb{F L}}\left(2 n, P_{2}^{\prime}, C-L\left(P_{1}^{\prime}\right)\right)$

(5) $M(n, P, C)=M\left(n, P_{1}^{\prime}, C\right) \cup M\left(n, P_{2}^{\prime}, C-L\left(P_{1}^{\prime}\right)\right)$ if $n=2^{k}$ for some $k \geq 0$

ACM Transactions on Programming Languages and Systems, Vol. TBD, No. TDB, Month Year. 
Proof. Note that for any derivation of $w^{\prime} \in \mathrm{P} \rightsquigarrow V$, the top is either of the form

$$
\frac{\frac{\cdots}{\left(w_{1}, w_{2}\right) \in \mathrm{P}_{1} \cdot \mathrm{P}_{3} \rightsquigarrow\left(V_{1}, V_{3}\right)}}{\frac{\left(w_{1}, w_{2}\right) \in\left(\mathrm{P}_{1}+\mathrm{P}_{2}\right) \cdot \mathrm{P}_{3} \rightsquigarrow\left(V_{1}+\mathrm{P}_{2}, V_{3}\right)}{w^{\prime}=w_{1} \cdot w_{2} \in \mathrm{P} \rightsquigarrow V=\left(V_{1}+\mathrm{P}_{2}\right) \cdot V_{3}}} \text { COR1 }
$$

or of the form

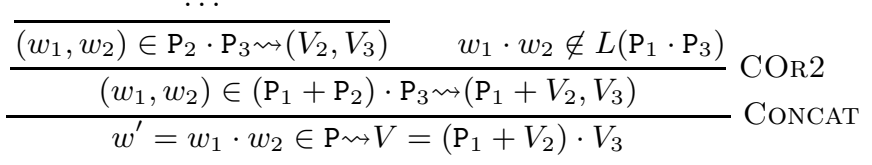

It is easily seen that hence $w^{\prime} \in \mathrm{P} \rightsquigarrow\left(V_{1}+\mathrm{P}_{2}\right) \cdot V_{3}$ iff $w^{\prime} \in \mathrm{P}_{1}^{\prime} \rightsquigarrow V_{1} \cdot V_{3}$ and that $w^{\prime} \in \mathrm{P} \rightsquigarrow\left(\mathrm{P}_{1}+V_{2}\right) \cdot V_{3}$ iff $w^{\prime} \in \mathrm{P}_{2}^{\prime} \rightsquigarrow V_{2} \cdot V_{3}$ and $w^{\prime} \notin L\left(\mathrm{P}_{1}^{\prime}\right)$. From these observations, equality (1) readily follows:

$$
\begin{aligned}
w \in \mathcal{T}^{\mathbb{F L}}(1, \mathrm{P}, C) \Leftrightarrow & \exists w^{\prime} \in C: w^{\prime} \in \mathrm{P} \rightsquigarrow V \wedge V(1)=w \\
\Leftrightarrow & \exists w^{\prime} \in C: w^{\prime} \in \mathrm{P} \rightsquigarrow\left(V_{1}+\mathrm{P}_{2}\right) \cdot V_{3} \wedge V_{1}(\lambda)=w \\
& \text { or } w^{\prime} \in \mathrm{P} \rightsquigarrow\left(\mathrm{P}_{1}+V_{2}\right) \cdot V_{3} \wedge V_{2}(\lambda)=w \\
\Leftrightarrow & \exists w^{\prime} \in C: w^{\prime} \in \mathrm{P}_{1}^{\prime} \rightsquigarrow V_{1} \cdot V_{3} \wedge V_{1}(\lambda)=w \\
& \text { or } w^{\prime} \in \mathrm{P}_{2}^{\prime} \rightsquigarrow V_{2} \cdot V_{3} \wedge V_{2}(\lambda)=w \wedge w^{\prime} \notin L\left(\mathrm{P}_{1}^{\prime}\right) \\
\Leftrightarrow & w \in \mathcal{T}^{\mathbb{F L}}\left(1, \mathrm{P}_{1}^{\prime}, C\right) \text { or } w \in \mathcal{T}^{\mathbb{F L}}\left(2, \mathrm{P}^{\prime}, C-L\left(\mathrm{P}_{1}^{\prime}\right)\right)
\end{aligned}
$$

Equalities (4) and (5) can be proven similarly. Note that, if $w^{\prime} \in \mathrm{P} \rightsquigarrow V$ and $V(11 n) \neq \perp$, then the matching derivation must be of the first form. Hence:

$$
\begin{aligned}
w \in \mathcal{T}^{\mathbb{F L}}(1, \mathrm{P}, C) & \Leftrightarrow \exists w^{\prime} \in C: w^{\prime} \in \mathrm{P} \rightsquigarrow V \wedge V(11 n)=w \\
& \Leftrightarrow \exists w^{\prime} \in C: w^{\prime} \in \mathrm{P} \rightsquigarrow\left(V_{1}+\mathrm{P}_{2}\right) \cdot V_{3} \wedge V_{1}(n)=w \\
& \Leftrightarrow \exists w^{\prime} \in C: w^{\prime} \in \mathrm{P}_{1}^{\prime} \rightsquigarrow V_{1} \cdot V_{3} \wedge V_{1}(\lambda)=w \\
& \Leftrightarrow w \in \mathcal{T}^{\mathbb{F L}}\left(1, \mathrm{P}_{1}^{\prime}, C\right)
\end{aligned}
$$

Equality (3) can be proven similarly.

Lemma 7.10. If $\left(w_{1}, w_{2}\right) \in P_{1} \cdot P_{2} \rightsquigarrow\left(V_{1}, V_{2}\right)$ then $w_{2} \in P_{2} \rightsquigarrow V_{2}$

Proof. The proof goes by induction on the matching derivation $\left(w_{1}, w_{2}\right) \in$ $\mathrm{P}_{1} \cdot \mathrm{P}_{2} \rightsquigarrow\left(V_{1}, V_{2}\right)$ with a case analysis on the last rule used. In all the cases, the result either follows immediately from the premise of the last rule used, or follows immediately from the induction hypothesis.

Proposition 7.11. If $P=\left(P_{1} \cdot P_{2}\right) \cdot P_{3}$ and $P^{\prime}=P_{1} \cdot\left(P_{2} \cdot P_{3}\right)$, then the following equalities hold:

(1) $\mathcal{T}^{\mathbb{E L}}(1, P, C)=M(\lambda, P, C) \backslash\left(\{\square\} \cdot \Sigma^{*}\right)$

(2) $\mathcal{T}^{\mathbb{F L}}(11 n, P, C)=\mathcal{T}^{\mathbb{F L}}\left(1 n, P^{\prime}, C\right)$

(3) $\mathcal{T}^{\mathbb{F L}}(12 n, P, C)=\mathcal{T}^{\mathbb{R L}}\left(21 n, P^{\prime}, C\right)$

(4) $\mathcal{T}^{\mathbb{F L}}(2 n, P, C)=\mathcal{T}^{\mathbb{F L}}\left(22 n, P^{\prime}, C\right)$

(5) $M(\lambda, P, C)=$ redistrib $\left(M\left(\lambda, P^{\prime}, C\right), M\left(2, P^{\prime}, C\right)\right)$

ACM Transactions on Programming Languages and Systems, Vol. TBD, No. TDB, Month Year. 
(6) $M(2 n, P, C)=M\left(22 n, P^{\prime}, C\right)$

Proof. We start by stating the following properties of the matching derivations of $\mathrm{P}$ and $\mathrm{P}^{\prime}$ :

(A) If $w^{\prime} \in \mathrm{P} \rightsquigarrow V$ then $V=\left(V_{1} \cdot V_{2}\right) \cdot V_{3}$ and $V_{3}(\lambda) \neq \perp$.

(B) If $w^{\prime} \in \mathrm{P}^{\prime} \rightsquigarrow V^{\prime}$ then $V^{\prime}=V_{1} \cdot\left(V_{2} \cdot V_{3}\right)$.

(C) $w^{\prime} \in \mathrm{P} \rightsquigarrow\left(V_{1} \cdot V_{2}\right) \cdot V_{3}$ iff $w^{\prime} \in \mathrm{P}^{\prime} \rightsquigarrow V_{1} \cdot\left(V_{2} \cdot V_{3}\right)$.

Property (A) holds because the top of every matching derivation of $w^{\prime} \in \mathrm{P} \rightsquigarrow V$ must look like:

$\frac{\frac{\cdots}{\frac{\left(w_{1}, w_{2} w_{3}\right) \in \mathrm{P}_{1} \cdot\left(\mathrm{P}_{2} \cdot \mathrm{P}_{3}\right) \rightsquigarrow\left(V_{1}, W\right)}{\left(w_{1} w_{2}, w_{3}\right) \in\left(\mathrm{P}_{1} \cdot \mathrm{P}_{2}\right) \cdot \mathrm{P}_{3} \rightsquigarrow\left(V_{1} \cdot V_{2}, V_{3}\right)} \quad \frac{\cdots}{\left(w_{2}, w_{3}\right) \in \mathrm{P}_{2} \cdot \mathrm{P}_{3} \rightsquigarrow\left(V_{2}, V_{3}\right)}}}{w^{\prime}=w_{1} w_{2} w_{3} \in\left(\mathrm{P}_{1} \cdot \mathrm{P}_{2}\right) \cdot \mathrm{P}_{3} \rightsquigarrow V=\left(V_{1} \cdot V_{2}\right) \cdot V_{3}}$ CCON

By application of Lemma 7.10 on $\left(w_{2}, w_{3}\right) \in \mathrm{P}_{2} \cdot \mathrm{P}_{3} \rightsquigarrow\left(V_{2}, V_{3}\right)$ we have $w_{3} \in \mathrm{P}_{3} \rightsquigarrow V_{3}$. Then $V_{3}(\lambda)=w_{3} \neq \perp$ by Lemma 7.4.

Property (B) holds because the top of every matching derivation of $w^{\prime} \in \mathrm{P}^{\prime} \rightsquigarrow V^{\prime}$ must look like:

$$
\frac{\overline{\left(w_{1}, w_{2} w_{3}\right) \in \mathrm{P}_{1} \cdot\left(\mathrm{P}_{2} \cdot \mathrm{P}_{3}\right) \rightsquigarrow\left(V_{1}, W\right)}}{w^{\prime}=w_{1} w_{2} w_{3} \in \mathrm{P}_{1} \cdot\left(\mathrm{P}_{2} \cdot \mathrm{P}_{3}\right) \rightsquigarrow V^{\prime}=V_{1} \cdot W} \text { CONCAT }
$$

By application of Lemma 7.10 on $\left(w_{1}, w_{2} w_{3}\right) \in \mathrm{P}_{1} \cdot\left(\mathrm{P}_{2} \cdot \mathrm{P}_{3}\right) \rightsquigarrow\left(V_{1}, W\right)$ we have $w_{2} \cdot w_{3} \in \mathrm{P}_{2} \cdot \mathrm{P}_{3} \rightsquigarrow W$. This derivation must end with an application of rule CoNCAT, so there must be a derivation of $\left(w_{2}, w_{3}\right) \in \mathrm{P}_{2} \cdot \mathrm{P}_{3} \rightsquigarrow\left(V_{2}, V_{3}\right)$ for some $V_{2}, V_{3}$ with $W=V_{2} \cdot V_{3}$. Hence, $V^{\prime}$ is of the form $V_{1} \cdot\left(V_{2} \cdot V_{3}\right)$.

To prove property $(\mathrm{C})$, suppose that $w^{\prime} \in \mathrm{P} \rightsquigarrow V$. We then have $\left(w_{1}, w_{2} w_{3}\right) \in$ $\mathrm{P}_{1} \cdot\left(\mathrm{P}_{2} \cdot \mathrm{P}_{3}\right) \rightsquigarrow\left(V_{1}, W\right)$ and $\left(w_{2}, w_{3}\right) \in \mathrm{P}_{2} \cdot \mathrm{P}_{3} \rightsquigarrow\left(V_{2}, V_{3}\right)$. Hence $w_{2} w_{3} \in \mathrm{P}_{2} \cdot \mathrm{P}_{3} \rightsquigarrow W$ by application of Lemma 7.10. Furthermore, $w_{2} w_{3} \in \mathrm{P}_{2} \cdot \mathrm{P}_{3} \rightsquigarrow V_{2} \cdot V_{3}$ by application of rule CONCAT on $\left(w_{2}, w_{3}\right) \in \mathrm{P}_{2} \cdot \mathrm{P}_{3} \rightsquigarrow\left(V_{2}, V_{3}\right)$. Hence, $W=V_{2} \cdot V_{3}$ by Theorem 6.4. Finally, $w^{\prime} \in \mathrm{P}^{\prime} \rightsquigarrow V_{1} \cdot\left(V_{2} \cdot V_{3}\right)$ by application of rule ConCAT on $\left(w_{1}, w_{2} w_{3}\right) \in \mathrm{P}_{1} \cdot\left(\mathrm{P}_{2} \cdot \mathrm{P}_{3}\right) \rightsquigarrow\left(V_{1}, V_{2} \cdot V_{3}\right)$. Conversely, suppose that $w^{\prime} \in \mathrm{P}^{\prime} \rightsquigarrow V^{\prime}$. By a reasoning similar to the one used to prove property (B) we obtain that $\left(w_{1}, w_{2} w_{3}\right) \in \mathrm{P}_{1} \cdot\left(\mathrm{P}_{2} \cdot \mathrm{P}_{3}\right) \rightsquigarrow\left(V_{1}, V_{2} \cdot V_{3}\right)$ and $w_{2} w_{3} \in \mathrm{P}_{2} \cdot \mathrm{P}_{3} \rightsquigarrow V_{2} \cdot V_{3}$. By application of rule CCON on these subderivations we obtain $\left(w_{1} w_{2}, w_{3}\right) \in \mathrm{P} \rightsquigarrow\left(V_{1} \cdot V_{2}, V_{3}\right)$. Finally, $w^{\prime} \in \mathrm{P} \rightsquigarrow\left(V_{1} \cdot V_{2}\right) \cdot V_{3}$ by application of rule ConcAT.

From property (A) equality (1) readily follows:

$$
\begin{aligned}
w \in \mathcal{T}^{\mathbb{L} L}(1, \mathrm{P}, C) & \Leftrightarrow \exists w^{\prime} \in C: w^{\prime} \in \mathrm{P} \rightsquigarrow V \wedge V(1)=w \\
& \Leftrightarrow \exists v \exists w^{\prime} \in C: w^{\prime} \in \mathrm{P} \rightsquigarrow V \wedge V(1)=w \wedge V(2)=v \\
& \Leftrightarrow \exists v: w \square v \in M(\lambda, \mathrm{P}, C) \\
& \Leftrightarrow w \in M(\lambda, \mathrm{P}, C) /\left(\{\square\} \cdot \Sigma^{*}\right)
\end{aligned}
$$


From all three properties equality (2) readily follows:

$$
\begin{aligned}
w \in \mathcal{T}^{\mathbb{R L}}(11 n, \mathrm{P}, C) & \Leftrightarrow \exists w^{\prime} \in C: w^{\prime} \in \mathrm{P} \rightsquigarrow V \wedge V(11 n)=w \\
& \Leftrightarrow \exists w^{\prime} \in C: w^{\prime} \in \mathrm{P} \rightsquigarrow\left(V_{1} \cdot V_{2}\right) \cdot V_{3} \wedge V_{1}(n)=w \\
& \Leftrightarrow \exists w^{\prime} \in C: w^{\prime} \in \mathrm{P}^{\prime} \rightsquigarrow V_{1} \cdot\left(V_{2} \cdot V_{3}\right) \wedge V_{1}(n)=w \\
& \Leftrightarrow w \in \mathcal{T}^{\mathbb{F L}}\left(1 n, \mathrm{P}^{\prime}, C\right)
\end{aligned}
$$

Equalities (3), (4), and (6) can be proven similarly. Let us abbreviate $M\left(\lambda, \mathrm{P}^{\prime}, C\right)$ by $M_{1}$ and $M\left(2, \mathrm{P}^{\prime}, C\right)$ by $M_{2}$. To prove equality (5) we observe:

$$
\begin{aligned}
v \square w_{3} \in & M(\lambda, \mathrm{P}, C) \\
\Leftrightarrow & \exists w^{\prime} \in C: w^{\prime} \in \mathrm{P} \rightsquigarrow V \wedge V(1)=v \wedge V(2)=w_{3} \\
\Leftrightarrow & \exists w^{\prime} \in C: w^{\prime} \in \mathrm{P} \rightsquigarrow\left(V_{1} \cdot V_{2}\right) \cdot V_{3} \wedge\left(V_{1} \cdot V_{2}\right)(\lambda)=v \wedge V_{3}(\lambda)=w_{3} \\
\Leftrightarrow & \exists w_{1}, w_{2} \exists w^{\prime} \in C: w^{\prime} \in \mathrm{P} \rightsquigarrow\left(V_{1} \cdot V_{2}\right) \cdot V_{3} \wedge w_{1} w_{2}=v \\
& \wedge V_{1}(\lambda)=w_{1} \wedge V_{2}(\lambda)=w_{2} \wedge V_{3}(\lambda)=w_{3} \\
\Leftrightarrow & \exists w_{1}, w_{2} \exists w^{\prime} \in C: w^{\prime} \in \mathrm{P}^{\prime} \rightsquigarrow V_{1} \cdot\left(V_{2} \cdot V_{3}\right) \wedge w_{1} w_{2}=v \\
& \wedge V_{1}(\lambda)=w_{1} \wedge V_{2}(\lambda)=w_{2} \wedge V_{3}(\lambda)=w_{3}
\end{aligned}
$$

We claim that the latter holds iff

$$
\exists w_{1}, w_{2}: w_{1} w_{2}=v \wedge w_{1} \square w_{2} w_{3} \in M_{1} \wedge w_{2} \square w_{3} \in M_{2},
$$

i.e., iff $v \square w_{3} \in$ redistrib $\left(M_{1}, M_{2}\right)$. The "only if" direction is obvious. To prove the "if" direction, let us assume $w_{1} \square w_{2} w_{3} \in M_{1}$ and $w_{2} \square w_{3} \in M_{2}$. By definition of $M_{1}$ and by property (B) there exists some $w^{\prime} \in C$ with $w^{\prime} \in \mathrm{P}^{\prime} \rightsquigarrow V_{1} \cdot\left(V_{2} \cdot V_{3}\right)$ such that $V_{1}(\lambda)=w_{1}$ and $\left(V_{2} \cdot V_{3}\right)(\lambda)=w_{2} w_{3}$. Then, by Lemma 7.4:

$$
w^{\prime}=\left(V_{1} \cdot\left(V_{2} \cdot V_{3}\right)\right)(\lambda)=V_{1}(\lambda) \cdot\left(V_{2} \cdot V_{3}\right)(\lambda)=w_{1} w_{2} w_{3} .
$$

Furthermore, since the derivation of $w^{\prime} \in \mathrm{P}^{\prime} \rightsquigarrow V_{1} \cdot\left(V_{2} \cdot V_{3}\right)$ must end with an application of rule ConcAT, we have $\left(w_{1}, w_{2} w_{3}\right) \in \mathrm{P}^{\prime} \rightsquigarrow\left(V_{1}, V_{2} \cdot V_{3}\right)$. Hence, $w_{2} w_{3} \in$ $\mathrm{P}_{2} \cdot \mathrm{P}_{3} \rightsquigarrow V_{2} \cdot V_{3}$ by Lemma 7.10 . Since $w_{2} \square w_{3} \in M_{2}$ we have by definition of $M_{2}$ and property (B) that there must exist some $w^{\prime \prime} \in C$ with $w^{\prime \prime} \in \mathrm{P}^{\prime} \rightsquigarrow V_{1}^{\prime} \cdot\left(V_{2}^{\prime} \cdot V_{3}^{\prime}\right)$, $V_{2}^{\prime}(\lambda)=w_{2}$, and $V_{3}^{\prime}(\lambda)=w_{3}$. Since the derivation of $w^{\prime \prime} \in \mathrm{P}^{\prime} \rightsquigarrow V_{1}^{\prime} \cdot\left(V_{2}^{\prime} \cdot V_{3}^{\prime}\right)$ must end with an application of rule CONCAT, we have $\left(w_{1}^{\prime \prime}, w_{2}^{\prime \prime} w_{3}^{\prime \prime}\right) \in \mathrm{P}^{\prime} \rightsquigarrow\left(V_{1}^{\prime}, V_{2}^{\prime} \cdot V_{3}^{\prime}\right)$ for $w_{1}^{\prime \prime} w_{2}^{\prime \prime} w_{3}^{\prime \prime}=w^{\prime \prime}$. Hence $w_{2}^{\prime \prime} w_{3}^{\prime \prime} \in \mathrm{P}_{2} \cdot \mathrm{P}_{3} \rightsquigarrow V_{2}^{\prime} \cdot V_{3}^{\prime}$ by Lemma 7.10. Furthermore, by Lemma 7.4 :

$$
w_{2}^{\prime \prime} w_{3}^{\prime \prime}=\left(V_{2}^{\prime} \cdot V_{3}^{\prime}\right)(\lambda)=V_{2}^{\prime}(\lambda) \cdot V_{3}^{\prime}(\lambda)=w_{2} w_{3} .
$$

Since we now have $w_{2} w_{3} \in \mathrm{P}_{2} \cdot \mathrm{P}_{3} \rightsquigarrow V_{2} \cdot V_{3}$ and $w_{2} w_{3} \in \mathrm{P}_{2} \cdot \mathrm{P}_{3} \rightsquigarrow V_{2}^{\prime} \cdot V_{3}^{\prime}$, we obtain $V_{2} \cdot V_{3}=V_{2}^{\prime} \cdot V_{3}^{\prime}$ by Theorem 6.4. Hence we have $w^{\prime} \in \mathrm{P}^{\prime} \rightsquigarrow V_{1} \cdot\left(V_{2} \cdot V_{3}\right)$ with $V_{1}(\lambda)=w_{1}, V_{2}(\lambda)=w_{2}$, and $V_{3}(\lambda)=w_{3}$.

\section{REGULAR HEDGE EXPRESSION PATTERNS}

The true power of regular expression pattern matching comes into play when we introduce regular hedge expression patterns matching hedges. A hedge is a sequence of trees; hedges form the basic data model of XML [Murata 1999; Vianu 2001]. In this section we formally define hedges, regular hedge languages, and regular hedge expression patterns. 
A hedge over $\Sigma$ is a sequence $\sigma_{1}\left[h_{1}\right] \ldots \sigma_{n}\left[h_{n}\right]$ where $n \geq 0, \sigma_{1}, \ldots, \sigma_{n}$ are symbols in $\Sigma$, and $h_{1}, \ldots, h_{n}$ are already hedges. The hedge with $n=0$ is called the empty hedge and will be denoted by $\lambda$. Hedges with $n=1$ are called trees. Hedges over $\Sigma$ will be denoted by $h, g$, and their subscripted versions. Note that if $h$ and $g$ are hedges, then so is $h g$, the concatenation of $h$ and $g$. Hedges of the form $\sigma[\lambda]$ will sometimes be abbreviated by $\sigma$.

Note that we cannot exactly model the trees in Figures 1(a) and 1(b) unless we put the actual data values ("Data On The Web", "Abiteboul", etc.) in the alphabet $\Sigma$. Putting all possible data values in our alphabet however, would result in an infinite alphabet. We will therefore abstract away from actual data values in pattern matching and assume $\Sigma$ to contain a special element data, for which we will replace all data values. The tree of Figure 1(a) then corresponds to

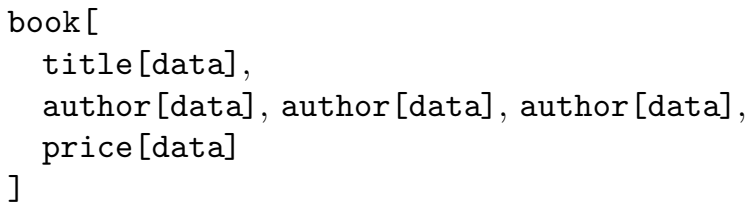

An actual programming language would provide features to retrieve the content of data nodes.

Just as regular word languages are defined as those languages that can be recognized by a finite word automaton, regular hedge languages are those languages that can be recognized by a finite hedge automaton [Brüggemann-Klein et al. 2001; Neven 2002]. A finite hedge automaton $H$ over $\Sigma$ is a tuple $(Q, \delta, F)$ where $Q$ is a finite set of states; $F$ is a regular language over $Q$; and $\delta$ is the transition relation: a possibly infinite set of triples $(q, \sigma, w)$ with $q \in Q$ and $w$ a word over $Q$, such for any $q$ and $\sigma$ the set $\{w \mid(q, \sigma, w) \in \delta\}$ is regular. We will denote this latter set by $\delta(q, \sigma)$. Since regular word languages are finitely representable by finite automata or regular expressions, the transition relation is also finitely representable. We associate a function $\delta^{*}$ with $\delta$ as follows: $\delta^{*}(\lambda)=\{\lambda\}$ and if $h=\sigma_{1}\left[h_{1}\right] \cdots \sigma_{n}\left[h_{n}\right]$ then

$$
\delta^{*}(h)=\left\{q_{1} \cdots q_{n} \mid \delta\left(q_{1}, \sigma_{1}\right) \cap \delta^{*}\left(h_{1}\right) \neq \emptyset, \ldots, \delta\left(q_{n}, \sigma_{n}\right) \cap \delta^{*}\left(h_{n}\right) \neq \emptyset\right\} .
$$

A hedge $h$ is accepted by a hedge automaton $H$ if $\delta^{*}(h) \cap F \neq \emptyset$. The language $L(H)$ recognized by a hedge automaton $H$ is the set of all hedges it accepts. A hedge language is regular if there exists some hedge automaton recognizing it. If $F \subseteq Q$ then $H$ can only accept trees, and $H$ is called a finite tree automaton. Its language is called a regular tree language. A hedge automaton is called total if $\delta^{*}(h) \neq \emptyset$ for all hedges $h$. Intuitively, a hedge automaton is total if it never gets "stuck" on any input. We can always make a hedge automaton total by adding a "garbage" state.

We will introduce regular hedge expression patterns next. While XDuce uses recursive patterns that allow the binding of nodes to subhedges which are arbitrarily deep in the input hedge, we will follow $\mathbb{C}$ Duce in the sense that we only allow to bind subhedges up to a certain depth. This will make the formalization considerably simpler. We still want our patterns to be able to recognize all regular hedge languages however, which can contain arbitrarily deep hedges. We therefore 
assume to be given a fixed set $\mathcal{N}$ of names, together with an environment $\Delta$. The set of names is assumed to be disjoint from $\Sigma$ and does not contain the special symbols $\perp$ and $\square$. The environment is a total function which relates every name $N \in \mathcal{N}$ with a regular tree language $\Delta(N)$. We will denote members of $\mathcal{N}$ by $N$, $M$ and their subscripted versions.

A regular expression hedge pattern $\mathrm{P}$ is an expression of the form $\varepsilon, N, \sigma\left[\mathrm{P}_{1}\right]$, $\mathrm{P}_{1}+\mathrm{P}_{2}, \mathrm{P}_{1} \cdot \mathrm{P}_{2}$, or $\mathrm{P}_{1}^{*}$ where $\mathrm{P}_{1}$ and $\mathrm{P}_{2}$ are already hedge patterns. The hedge language $L(\mathrm{P})$ of a hedge pattern $\mathrm{P}$ is defined as follows:

$$
\begin{array}{ll}
L(\varepsilon) & =\{\lambda\} \\
L(\sigma[\mathrm{P}]) & =\{\sigma[h] \mid h \in L(\mathrm{P})\} \\
L(N) & =\Delta(N) \\
L\left(\mathrm{P}_{1}+\mathrm{P}_{2}\right) & =L\left(\mathrm{P}_{1}\right) \cup L\left(\mathrm{P}_{2}\right) \\
L\left(\mathrm{P}_{1} \cdot \mathrm{P}_{2}\right) & =L\left(\mathrm{P}_{1}\right) \cdot L\left(\mathrm{P}_{2}\right) \\
L\left(\mathrm{P}^{*}\right) & =L(\mathrm{P})^{*}
\end{array}
$$

It is easy to see that $L(\mathrm{P})$ is always regular. As in Section 3, we identify $\mathrm{P}$ with the partial function $\mathrm{P}:\{1,2\}^{*} \rightarrow\{\varepsilon,+, \cdot, *\} \cup \Sigma \cup \mathcal{N}$ such that:

-if $\mathrm{P}=\varepsilon$ then $\operatorname{dom}(\mathrm{P})=\{\lambda\}$ and $\mathrm{P}(\lambda)=\varepsilon$;

-if $\mathrm{P}=N$ with $N \in \mathcal{N}$ then $\operatorname{dom}(\mathrm{P})=\{\lambda\}$ and $\mathrm{P}(\lambda)=N$;

-if $\mathrm{P}=\sigma\left[\mathrm{P}_{1}\right]$ with $\sigma \in \Sigma$ then $\operatorname{dom}(\mathrm{P})=\{\lambda\} \cup\left\{1 n \mid n \in \operatorname{dom}\left(\mathrm{P}_{1}\right)\right\}$ with $\mathrm{P}(\lambda)=\sigma$ and $\mathrm{P}(1 n)=\mathrm{P}_{1}(n)$;

- if $\mathrm{P}=\mathrm{P}_{1}^{*}$ then we make a similar definition, only $\mathrm{P}(\lambda)=*$;

-if $\mathrm{P}=\mathrm{P}_{1}+\mathrm{P}_{2}$ then $\operatorname{dom}(\mathrm{P})=\{\lambda\} \cup\left\{1 n \mid n \in \operatorname{dom}\left(\mathrm{P}_{1}\right)\right\} \cup\left\{2 n \mid n \in \operatorname{dom}\left(\mathrm{P}_{2}\right)\right\}$ with $\mathrm{P}(\lambda)=+, \mathrm{P}(1 n)=\mathrm{P}_{1}(n)$, and $\mathrm{P}(2 n)=\mathrm{P}_{2}(n)$; and

-if $\mathrm{P}=\mathrm{P}_{1} \cdot \mathrm{P}_{2}$ we make a similar definition, only $\mathrm{P}(\lambda)=$.

Precedence of operators is the same as in Section 3. As before, the set of bindable nodes $b n(\mathrm{P})$ of a hedge pattern $\mathrm{P}$ are those nodes in its domain which do not have an ancestor node labeled with $*$.

We will use $\sigma[V]$ to denote the association function with domain $\{\lambda\} \cup\{1 n \mid n \in$ $\operatorname{dom}(V)\}$ such that $(\sigma[V])(\lambda)=\sigma[V(\lambda)]$ and $(\sigma[V])(1 n)=V(1 n)$.

In XDuce two kinds of patterns were introduced: external patterns which largely correspond to the hedge patterns introduced above and internal patterns to which the external patterns are translated. The internal patterns are used to define the matching relation and to do type inference. These patterns can only recognize ranked trees, which are trees in which each label has a fixed number of children. It is therefore necessary to encode the unranked input trees (where a label can have an arbitrary number of children) into ranked trees before matching. We have chosen to work directly with the external, unranked, representation of patterns in this paper because our insights gained for regular string expression patterns can be directly extended to regular hedge expression patterns without using such internal patterns (as we will show in the following sections). This hugely simplifies the correctness proof of our type inference algorithm for hedges, as it largely follows from that of the algorithms given earlier. 


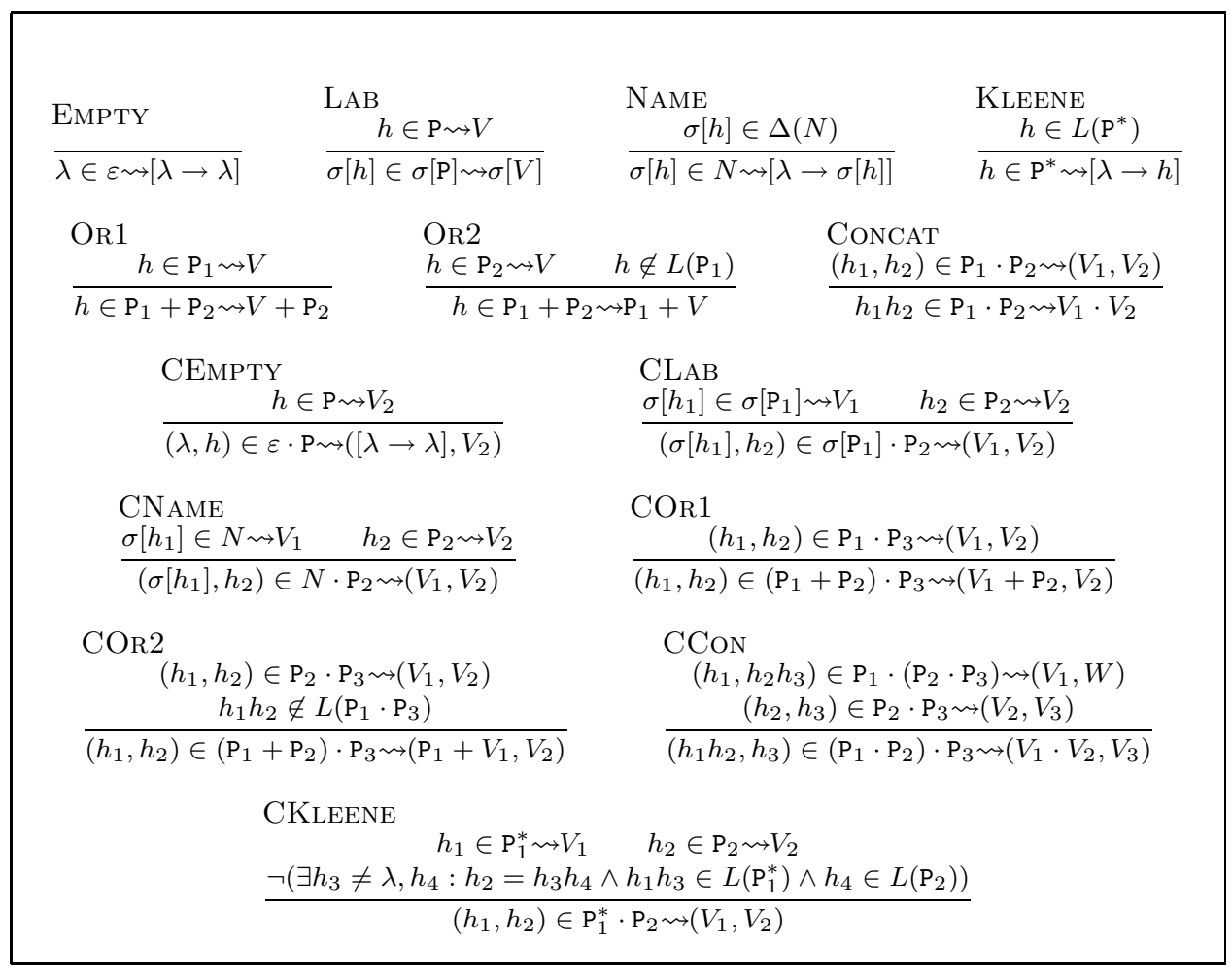

Fig. 5. The matching relation $h \in \mathrm{P} \rightsquigarrow V$ for hedges under the first and longest match disambiguation policy.

\section{HEDGE MATCHING UNDER THE FIRST AND LONGEST MATCH POLICY}

In this section we lift the matching process under the first and longest match policy to hedges. Its associated type inference problem will be solved in the following section. We can lift the matching process and type inference algorithm for the POSIX policy in a similar way.

The matching relation for hedge regular expressions under the first and longest match policy is defined in Figure 5. Most of the rules are simple extensions to hedges of the rules in Figure 4. For example, rule LAB now allows us to match hedge $\sigma[h]$ against pattern $\sigma[\mathrm{P}]$ if $h$ can be matched against P. Note that if we view a word $\sigma_{1} \ldots \sigma_{n}$ as a hedge $\sigma_{1}[\lambda] \ldots \sigma_{n}[\lambda]$, we get exactly the semantics of Figure 4 . There are only two rules not occurring in the word case: NAME and CNAmE. Rule NAmE states that a tree is matched by a name $N$ if the tree belongs to the associated tree language $\Delta(N)$. Rule CNAME is similar, but is used in concatenations.

The following theorem is the equivalent of Theorem 6.4:

THEOREM 9.1. The matching relation of Figure 5 is well defined:

(1) The matching relation is semantically correct: $h \in P \rightsquigarrow V$ iff $h \in L(P)$, and,

(2) The matching relation is unique: if $h \in P \rightsquigarrow V$ and $h \in P \rightsquigarrow W$ then $V=W$.

Proof. Completely analogous to the proof of Theorem 6.4. 


\section{TYPE INFERENCE FOR HEDGES UNDER THE FIRST AND LONGEST MATCH POLICY}

In this section we lift the type inference algorithm of Section 7 to the hedge setting. Concretely, we will show:

THEOREM 10.1. If $P$ is a hedge pattern and $C$ is a regular hedge language then $\mathcal{T}^{\mathbb{R L}}(n, P, C)$ is also regular, and can be effectively computed.

\subsection{The algorithm}

We obtain the type inference for hedges by a modification of Algorithm 2. Algorithm 2 uses quotient, breaking, and redistribution on word languages. The corresponding operations on hedge languages are defined in the obvious way. For example, the left quotient of hedge language $L$ by hedge language $K$, denoted as $K \backslash L$, is the set $\{s \mid \exists p \in K: p s \in L\}$.

The main observations we made for the word setting can be transfered in a straightforward manner to the hedge setting. For example, $\mathcal{T}^{\mathbb{F L}}(\lambda, \mathrm{P}, C)=L(\mathrm{P}) \cap C$ for any $\mathrm{P}$. Likewise, if $\mathrm{P}=\mathrm{P}_{1}+\mathrm{P}_{2}$ then $\mathcal{T}^{\mathbb{E L}}(1 n, \mathrm{P}, C)=\mathcal{T}^{\mathbb{F L}}\left(n, \mathrm{P}_{1}, C\right)$ and $\mathcal{T}^{\mathbb{F L}}(2 n, \mathrm{P}, C)=\mathcal{T}^{\mathbb{F L}}\left(n, \mathrm{P}_{2}, C-L\left(\mathrm{P}_{1}\right)\right)$. The case where $\mathrm{P}=\mathrm{P}_{1} \cdot \mathrm{P}_{2}$ with $\mathrm{P}_{1}=\varepsilon, \mathrm{P}_{1}=$ $N$ or $\mathrm{P}_{1}=\sigma\left[\mathrm{P}^{\prime}\right]$ can also be deduced using a reasoning similar to the word setting: $\mathcal{T}^{\mathbb{F L}}(1 n, \mathrm{P}, C)=\mathcal{T}^{\mathbb{F L}}\left(n, \mathrm{P}_{1}, C / L\left(\mathrm{P}_{2}\right)\right)$ and $\mathcal{T}^{\mathbb{R L}}(2 n, \mathrm{P}, C)=\mathcal{T}^{\mathbb{F L}}\left(n, \mathrm{P}_{2}, L\left(\mathrm{P}_{1}\right) \backslash C\right)$. The other cases can also be lifted to the hedge setting.

The only case when we cannot fall back on our insights of the word setting is when we need to calculate the type of $1 n$ in $\mathrm{P}=\sigma\left[\mathrm{P}_{1}\right]$. Intuitively, a hedge can only be associated to a subpattern of $\mathrm{P}_{1}$ in $\mathrm{P}=\sigma\left[\mathrm{P}_{1}\right]$, if it is a subhedge of a hedge $h$ matched by $\mathrm{P}_{1}$ such that $\sigma[h] \in C$. Hence, if we define the cut of a hedge language $L$ by a symbol $\sigma$, denoted by $\operatorname{cut}(L, \sigma)$, as $\{h \mid \sigma[h] \in L\}$ then $\mathcal{T}^{\mathbb{F L}}(1 n, \mathrm{P}, C)$ equals $\mathcal{T}^{\mathbb{F L}}\left(n, \mathrm{P}_{1}, \operatorname{cut}(C, \sigma)\right)$. Of course, we need to be able to calculate cuts:

Lemma 10.2. If $L$ is a regular hedge language, then so is $\operatorname{cut}(L, \sigma)$.

Proof. Since $L$ is a regular hedge language, there exists a finite hedge automaton $H=(Q, \delta, F)$ such that $L(H)=L$. Let $S=F \cap Q$. Intuitively, $S$ contains those states in $F$ the automaton can be in after processing a tree. We then define $F^{\prime}=\bigcup_{q \in S} \delta(q, \sigma)$ and $H^{\prime}=\left(Q, \delta, F^{\prime}\right)$. It is easy to see that $\sigma[h] \in L(H)$ iff $h \in H^{\prime}$. Hence $L\left(H^{\prime}\right)=\operatorname{cut}(L, \sigma)$.

The type inference algorithm for hedges is then obtained from Algorithm 2 by lifting all operations to the hedge setting, and adding the case for $m=1 n$ and $\mathrm{P}=\sigma\left[\mathrm{P}_{1}\right]$, as shown in Algorithm 4. The dots indicate the cases which are similar to the word setting.

\subsection{Proof of correctness}

Before we talk about the correctness of Algorithm 4, we need to show that the operations used are still computable for regular hedge languages.

It is well-known that hedge languages are closed under union, intersection and negation [Brüggemann-Klein et al. 2001]. It is also well-known that finite hedge languages are regular, as is the set of all hedges. Regular hedge languages are also closed under left and right quotient. Although the proof is straightforward, it has 


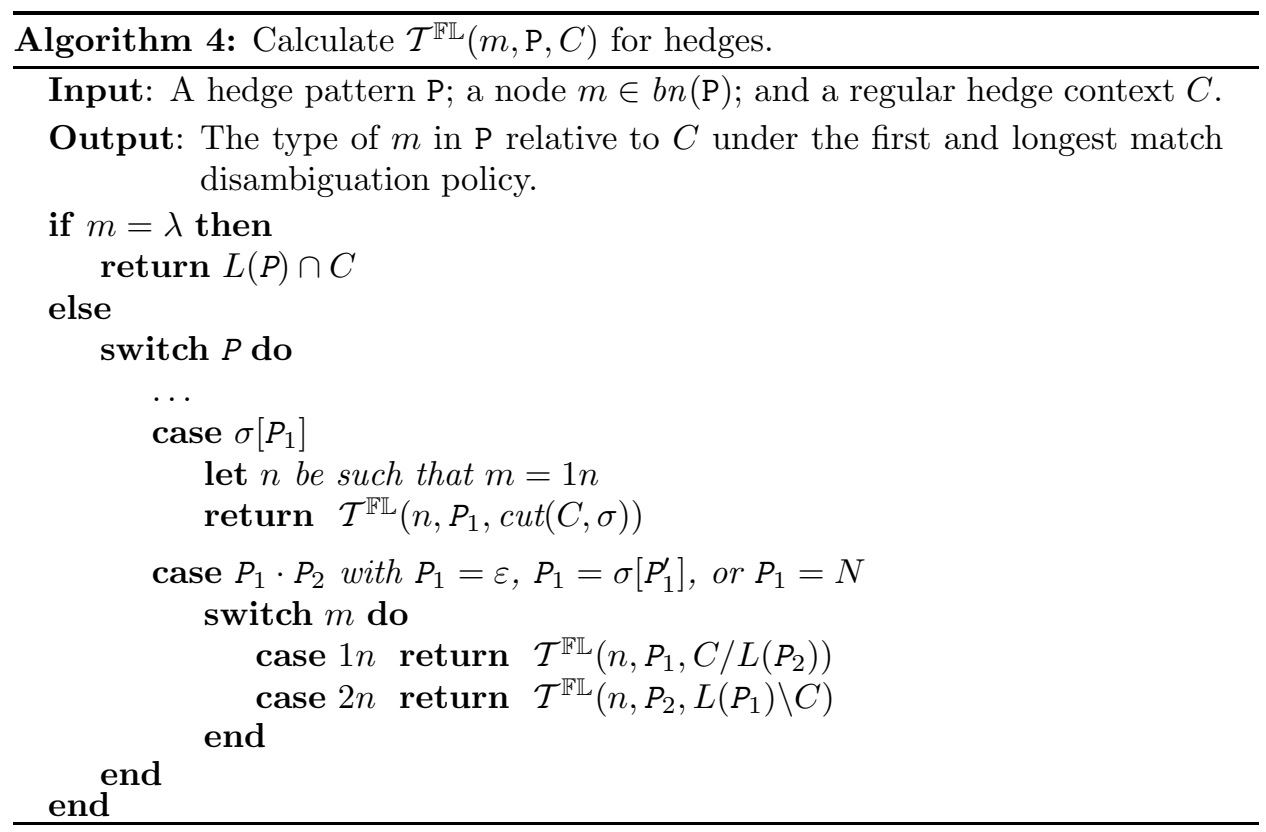

not yet been explicitly given in the literature. For the sake of completeness we therefore provide it in Appendix A.

If $L$ is a regular hedge language, then so is the language

$$
\pi^{-1}(L):=\left\{h_{1} \square h_{2} \square \cdots \square h_{n} \mid h_{1} h_{2} \ldots h_{n} \in L\right\} .
$$

Indeed, we can add the transition $(q, \square, \lambda)$ to an automaton $H=(Q, \delta, F)$ for $L$, where $q$ is a new state. It then suffices to allow the reading of $q$ at arbitrary places in $F$ (modify a DFA for $F$ to allow reading the letter $q$, which is then ignored). The closure of regular hedge languages under breakings then follows from Lemma 5.2.

Closure under redistribution follows by the following lemma:

Lemma 10.3. If $M_{1}$ and $M_{2}$ are regular marked hedge languages, then so is redistrib $\left(M_{1}, M_{2}\right)$, which can effectively be computed.

Proof. We introduce two operations on hedge languages:

$$
\begin{aligned}
\iota(L) & =\left\{h_{1} \square h_{2} \square h_{3} \mid h_{1} \square h_{2} h_{3} \in L\right\}, \\
\pi_{1}(L) & =\left\{h_{1} h_{2} \square h_{3} \mid h_{1} \square h_{2} \square h_{3} \in L\right\} .
\end{aligned}
$$

We will show that if $M$ is a marked hedge language then $\iota(M)$ is a regular hedge language and if $N$ is a regular hedge language containing only hedges of the form $h_{1} \square h_{2} \square h_{3}$ then $\pi_{1}(N)$ is a regular marked hedge language. The lemma then follows since:

$$
\text { redistrib }\left(M_{1}, M_{2}\right)=\pi_{1}\left(\iota\left(M_{1}\right) \cap\left(\mathcal{H}(\Sigma) \cdot\{\square\} \cdot M_{2}\right) .\right.
$$

Let $M$ be a marked regular hedge language and $H=(Q, \delta, F)$ a hedge automaton recognizing $M$. Since $M$ is a marked hedge language, every hedge in $M$ is of the form $h_{1} \square h_{2}$ where the symbol $\square$ does not occur in $h_{1}$ or $h_{2}$. Then every word in 
$F$ must be of the form $w_{1} q w_{2}$ with $\delta(q, \square)=\{\lambda\}$ and $\delta(q, \sigma)=\emptyset$ for all $\sigma$. If this would be not the case, we can find a hedge $h$ in $M$ not containing $\square$. We may then assume w.l.o.g. that there is exactly one such $q$ (if there are more, we can group them all together in one new state). Let us define the language

$$
\iota^{q}(F):=\left\{w_{1} q w_{2} q w_{3} \mid w_{1} q w_{2} w_{3} \in F\right\} .
$$

This is clearly a regular language: modify an automaton for $F$ such that an extra $q$ can be read after the first one. Define $R=\left(Q, \delta, \iota^{q}(F)\right)$. It is easy to see that $h_{1} \square h_{2} h_{3} \in L(H)$ iff $h_{1} \square h_{2} \square h_{3} \in L(R)$. Hence $L(R)=\iota(M)$.

Let $N$ be regular hedge language containing only hedges of the form $h_{1} \square h_{2} \square h_{3}$ and let $H=(Q, \delta, F)$ be a hedge automaton recognizing $N$. Then every word in $F$ must be of the form $w_{1} q w_{2} q^{\prime} w_{3}$ with $\delta(q, \square)=\delta\left(q^{\prime}, \square\right)=\{\lambda\}$ and $\delta(q, \sigma)=$ $\delta\left(q^{\prime}, \sigma\right)=\emptyset$ for all $\sigma$. If this would be not the case, we can find a hedge $h$ in $M$ not containing $\square$ or containing only one $\square$. We may assume that there is only one such $q$ and $q^{\prime}$ (if there are more we can group them all together in a new state). Then define

$$
\pi_{1}^{q}(F):=\left\{w_{1} w_{2} q w_{3} \mid w_{1} q w_{2} q^{\prime} w_{3} \in F\right\} .
$$

This is clearly a regular language: modify an automaton for $F$ to forget $q^{\prime}$. Define $R=\left(Q, \delta, \pi_{1}^{q}(F)\right)$. It is easy to see that $h_{1} \square h_{2} \square h_{3} \in L(H)$ iff $h_{1} h_{2} \square h_{3} \in L(R)$. Hence $L(R)=\pi_{1}(M)$.

The correctness of Algorithm 4 then follows from the fact that the propositions in Section 10.2 remain valid for the hedge setting, and the following two propositions:

Proposition 10.4. If $P=P_{1} \cdot P_{2}$, then the following equalities hold:

(1) $\mathcal{T}^{\mathbb{F L}}(1 n, P, C)=\mathcal{T}^{\mathbb{R L}}\left(n, P_{1}, C / L\left(P_{2}\right)\right)$

(2) $\mathcal{T}^{\mathbb{F L}}(2 n, P, C)=\mathcal{T}^{\mathbb{F L}}\left(n, P_{2},\left(L\left(P_{1}\right) \backslash C\right)\right)$

(3) $M(\lambda, P, C)=\mathcal{T}^{\mathbb{F L}}(1, P, C) \cdot\{\square\} \cdot \mathcal{T}^{\mathbb{F L}}(2, P, C)$

(4) $M(2 n, P, C)=M\left(n, P_{2}, L\left(P_{1}\right) \backslash C\right)$

Proof. Similar to that of Proposition 7.7.

Proposition 10.5. If $P=\sigma\left[P_{1}\right]$, then $\mathcal{T}^{\mathbb{R L}}(1 n, P, C)=\mathcal{T}^{\mathbb{F L}}\left(n, P_{1}, \operatorname{cut}(C, \sigma)\right)$

Proof. Every matching derivation $h^{\prime} \in \mathrm{P} \rightsquigarrow V$ must be of the form

$$
\frac{\frac{\cdots}{h_{1} \in \mathrm{P}_{1} \rightsquigarrow V_{1}}}{h^{\prime}=\sigma\left[h_{1}\right] \in \mathrm{P} \rightsquigarrow V=\sigma\left[V_{1}\right]} \text { LAB }
$$

The proposition readily follows:

$$
\begin{aligned}
h \in \mathcal{T}^{\mathbb{L}}(1 n, \mathrm{P}, C) & \Leftrightarrow \exists h^{\prime} \in C: h^{\prime} \in \mathrm{P} \rightsquigarrow V \wedge V(1 n)=h \\
& \Leftrightarrow \exists h_{1}: \sigma\left[h_{1}\right] \in C \wedge h_{1} \in \mathrm{P}_{1} \rightsquigarrow V_{1} \wedge V_{1}(n)=h \\
& \Leftrightarrow \exists h_{1} \in \operatorname{cut}(C, \sigma): h_{1} \in \mathrm{P}_{1} \rightsquigarrow V_{1} \wedge V_{1}(n)=h \\
& \Leftrightarrow h \in \mathcal{T}^{\mathbb{F L}}\left(n, \mathrm{P}_{1}, \operatorname{cut}(C, \sigma)\right)
\end{aligned}
$$




\section{DISCUSSION AND FUTURE WORK}

In this paper we have focussed on the longest match semantics in the POSIX and first and longest match disambiguation policies. One could also consider a shortest match disambiguation rule and even a mixture of longest and shortest match. Indeed, for the POSIX policy we could enrich the patterns with a shortest match concatenation operator, denoted by. ? The pattern $\mathrm{P}_{1}$ in $\mathrm{P}_{1} .{ }^{?} \mathrm{P}_{2}$ then matches as little of the input as possible, still allowing the rest of the pattern to match. Likewise, for the first and longest match policy we could enrich the patterns with a shortest match Kleene star operator, denoted by $*^{\text {? }}$. The matching relation and type inference algorithm presented here can be extended in a straightforward manner to include these operators.

We note that in languages such as sed and awk, regular expression patterns are not required to match all of their input. They just have to start matching as early as possible in the input string, and can stop as soon as a match is found. Using the shortest-match concatenation operator introduced above, we can simulate this behavior for the POSIX disambiguation policy by transforming P into $\Sigma^{*} . ? \mathrm{P} \cdot \Sigma^{*}$.

Whereas we restrict the bindable nodes of a pattern to those nodes not occurring in a Kleene closure, $\mathbb{C}$ Duce defines all nodes bindable, allowing patterns like:

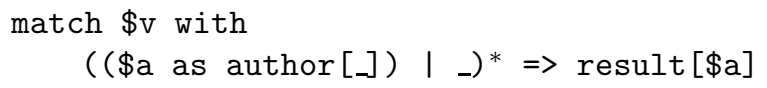

Here, every subhedge matched by author [] is concatenated to the value of $\$ \mathrm{a}$. The XDuce policy continues to be used inside the Kleene closure to disambiguate if necessary. It is not immediately clear how our type inference techniques can be adapted to this setting. POSIX also define all nodes bindable, where variables inside the Kleene closure get bound to the last value matched. Again, it is not immediately clear how to adapt our type inference algorithm to this setting.

In this paper, we have focused on gaining fundamental insights into the type inference problem for unique pattern matching, and have not concerned ourselves with the practical implementation of our algorithms. We have also not considered the associated time and space requirements. To our knowledge, there has not yet been a formal investigation of the inherent time complexity bounds of the regular type inference problem. These bounds may depend on the way regular languages are represented (i.e. as finite automata, as regular expressions, or yet other formalisms). We note that any type inference algorithm using non-deterministic finite automata to represent regular sets must have has at least an exponential worst case running time. Indeed, $\mathcal{T}^{\mathbb{P}}\left(2, \mathrm{P}+\Sigma^{*}, \Sigma^{*}\right)=\mathcal{T}^{\mathbb{R}}\left(2, \mathrm{P}+\Sigma^{*}, \Sigma^{*}\right)=\Sigma^{*}-L(\mathrm{P})$, the complement of $L(\mathrm{P})$. It is well-known that complementation of regular languages using nondeterministic automata can cause an exponential blow-up [Hopcroft and Ullman 1979].

As such, although in principle any finite (hedge) automaton library can be used to implement the type inference algorithms of this paper, it would be worthwhile to investigate which algorithms lend themselves to an acceptable performance in practice. A starting point here can be the work on MONA [Klarlund and Møller 2001; Elgaard et al. 1998], XDuce [Hosoya 2000; Hosoya et al. 2005], and $\mathbb{C D u c e}$ [Frisch et al. 2002]. 


\section{ACKNOWLEDGMENTS}

I thank the anonymous referees, Jan Van den Bussche, Dirk Leinders, Wim Martens, and Frank Neven for inspiring discussions and for their constructive comments on a draft version of this paper.

\section{REFERENCES}

Abiteboul, S., Quass, D., McHugh, J., Widom, J., And Wiener, J. L. 1997. The Lorel query language for semistructured data. International Journal on Digital Libraries 1, 1, 68-88.

BaAder, F. And Nipkow, T. 1998. Term Rewriting and All That. Cambridge University Press. Section 2.3.

Boag, S., Chamberlin, D., Fernández, M. F., Florescu, D., Robie, J., And Siméon, J. 2005. XQuery 1.0: An XML Query Language. W3C Working Draft.

Book, R., Even, S., Greibach, S., and Ott, G. 1971. Ambiguity in graphs and expressions. IEEE Transactions on Computers 20, 2, 149-153.

Brüggemann-Klein, A., Murata, M., And Wood, D. 2001. Regular tree and regular hedge languages over unranked alphabets. Unpublished manuscript, version 1.

Buneman, P., Fernandez, M. F., And Suciu, D. 2000. UnQL: a query language and algebra for semistructured data based on structural recursion. VLDB Journal: Very Large Data Bases 9, 1, $76-110$.

Clark, J. And Makoto, M. 2001. RELAX NG Specification. Organization for the Advancement of Structured Information Standards.

Davidson, A., Fuchs, M., Hedin, M., Jain, M., Koistinen, J., Lloyd, C., Maloney, M., And Schwarzhof, K. 1999. Schema for object-oriented XML 2.0. Tech. rep., Veo Systems Inc.

Dougherty, D. And Robbins, A. 1996. Sed and Awk. O'Reilly.

ElgaArd, J., Klarlund, N., ANd Møller, A. 1998. Mona 1.x: new techniques for WS1S and WS2S. In Computer Aided Verification, CAV '98, Proceedings. LNCS, vol. 1427. Springer Verlag.

FRISCH, A. 2004. Regular tree language recognition with static information. In Exploring New Frontiers of Theoretical Informatics, IFIP 18th World Computer Congress, TCS 3rd International Conference on Theoretical Computer Science. Kluwer, 661-674.

Frisch, A. AND Cardelli, L. 2004. Greedy regular expression matching. In Automata, Languages and Programming: ICALP 2004. Proceedings. Lecture Notes in Computer Science, vol. 3142. 618-629.

Frisch, A., Castagna, G., and Benzaken, V. 2002. Semantic subtyping. In Proceedings of the Seventeenth Annual IEEE Symposium on Logic in Computer Science. IEEE Computer Society Press, 137-146.

Frisch, A., Castagna, G., and Benzaken, V. 2003. CDuce: an XML-centric general-purpose language. In Proceedings of the eighth ACM SIGPLAN international conference on Functional programming. ACM Press, 51-63.

Hopcroft, J. E. And Ullman, J. D. 1979. Introduction to Automata Theory, Languages and Computation. Addison-Wesley.

Hosoya, H. 2000. Regular expression types for XML. Ph.D. thesis, University of Tokyo.

Hosoya, H. 2003. Regular expression pattern matching - a simpler design. Tech. Rep. 1397, RIMS, Kyoto University.

Hosoya, H. AND PIERCE, B. C. 2002. Regular expression pattern matching for XML. Journal of Functional Programming 13, 6, 961-1004.

Hosoya, H. And PIErce, B. C. 2003. XDuce: A statically typed XML processing language. ACM Transactions on Internet Technology (TOIT) 3, 2, 117-148.

Hosoya, H., Vouillon, J., And Pierce, B. C. 2005. Regular expression types for XML. ACM Transactions on Programming Languages and Systems 27, 1, 46-90.

Institute of Electrical and Electronic Engineers. 1992. Portable operating system interface (POSIX). IEEE Std 1003.2.

ACM Transactions on Programming Languages and Systems, Vol. TBD, No. TDB, Month Year. 
Klarlund, N. AND Møller, A. 2001. MONA Version 1.4 User Manual. Basic Research In Computer Science (BRICS) Notes Series NS-01-1, Department of Computer Science, University of Aarhus.

LAURIKARI, V. 2000. NFAs with tagged transitions, their conversion to deterministic automata and application to regular expressions. In Symposium on String Processing and Information Retrieval (SPIRE).

LAURIKARI, V. 2001. Efficient submatch addressing for regular expressions. M.S. thesis, Helsinki University of Technology.

LEvin, M. Y. 2003. Compiling regular patterns. In Proceedings of the eighth ACM SIGPLAN international conference on Functional programming. ACM Press, 65-77.

Møller, A. 2003. Document structure description 2.0. Tech. rep., Basic Research In Computer Science (BRICS), Department of Computer Science, University of Aarhus.

Murata, M. 1999. Hedge automata: a formal model for XML schemata. Available at http: //www.geocities.com/murata_makoto.

Murata, M. 2001. Extended path expressions for XML. In Proceedings of the twentieth ACM symposium on Principles of database systems. ACM Press, 126-137.

Murata, M., Lee, D., And Mani, M. 2001. Taxonomy of XML schema languages using formal language theory. In Extreme Markup Languages.

Neumann, A. And Seidl, H. 1998. Locating matches of tree patterns in forests. In Foundations of Software Technology and Theoretical Computer Science. LNCS 1530. 134-145.

Neven, F. 2002. Automata theory for XML researchers. ACM SIGMOD Record 31, 3, 39-46.

Neven, F. And Schwentick, T. 2001. Automata- and logic-based pattern languages for treestructured data. In Semantics in Databases. Vol. 2582. LNCS, Springer, 160-178.

Sterling, L. And Shapiro, E. 1994. The Art of Prolog (second edition). MIT Press.

SucIU, D. 2002. The XML typechecking problem. ACM SIGMOD Record 31, 1, 89-96.

Sumir, E. May 2003. Personal Communication.

Tabuchi, N., Sumit, E., And Yonezawa, A. 2002. Regular expression types for strings in a text processing language (extended abstract). In Workshop on Types in Programming (TIP'02). http://web.yl.is.s.u-tokyo.ac.jp/ tabee/xperl/.

Thompson, H. S., Beech, D., Maloney, M., and Mendelsohn, N. 2001. XML Schema. W3C Recommendation.

Ullman, J. D. 1998. Elements of ML Programming, Second ed. Prentice Hall.

Vianu, V. 2001. A web odyssey: from Codd to XML. In Proceedings of the twentieth ACM SIGMOD-SIGACT-SIGART symposium on Principles of database systems. ACM Press, 1-15.

Wall, L., Christiansen, T., And Orwant, J. 2000. Programming Perl, 3rd ed. O'Reilly \& Associates.

Yergeau, F., Bray, T., Paoli, J., Sperberg-McQueen, C. M., And Maler, E. 2004. Extensible Markup Language (XML) 1.0 (Third Edition). W3C Recommendation.

\section{A. CLOSURE OF REGULAR HEGDE LANGUAGES UNDER LEFT AND RIGHT QUOTIENTS}

To our knowledge, the closure of regular hedge languages under left and right quotients has not yet been explicitly proven in the literature. We therefore prove it here.

Lemma A.1. Regular hedge languages are closed under left and right quotient.

Proof. Assume that $L$ and $K$ are regular hedge languages. By definition, there exist hedge automata $H=\left(Q_{H}, \delta_{H}, F_{H}\right)$ and $G=\left(Q_{G}, \delta_{G}, F_{G}\right)$ such that $L(H)=L$ and $L(G)=K$. We assume without loss of generality that $H$ and $G$ are total.

We will now show how to construct a hedge automaton that recognizes $L / K$. Let $W_{1}$ be a regular word language over $Q_{H}$ and let $W_{2}$ be a regular word language over 
$Q_{G}$. We define the simultaneous product $W_{1} \times W_{2}$ of $W_{1}$ and $W_{2}$ as the language over $Q_{H} \times Q_{G}$ such that

$$
W_{1} \times W_{2}=\left\{\left(q_{1}, s_{1}\right) \cdots\left(q_{n}, s_{n}\right) \mid q_{1} \cdots q_{n} \in W_{1}, s_{1} \cdots s_{n} \in W_{2}\right\} .
$$

To prove the regularity of $W_{1} \times W_{2}$, let us define the following two word languages:

$$
\begin{aligned}
& \pi_{1}^{-1}\left(W_{1}\right)=\left\{\left(q_{1}, s_{1}\right) \cdots\left(q_{n}, s_{n}\right) \mid q_{1} \cdots q_{n} \in W_{1}, s_{j} \in Q_{G}\right\}, \\
& \pi_{2}^{-1}\left(W_{2}\right)=\left\{\left(q_{1}, s_{1}\right) \cdots\left(q_{n}, s_{n}\right) \mid s_{1} \cdots s_{n} \in W_{2}, q_{i} \in Q_{H}\right\} .
\end{aligned}
$$

It is clear that $\pi_{1}^{-1}\left(W_{1}\right)$ and $\pi_{2}^{-1}\left(W_{2}\right)$ are regular word languages over $Q_{H} \times Q_{G}$ (we can modify an automaton for $W_{1}$ or $W_{2}$ to allow reading symbols in $Q_{H} \times Q_{G}$, taking into account the original symbol to be read). Then $W_{1} \times W_{2}$ is also regular since $W_{1} \times W_{2}=\pi_{1}^{-1}\left(W_{1}\right) \cap \pi_{2}^{-1}\left(W_{2}\right)$.

Let $R=(Q, \delta, F)$ be the hedge automaton with

$-Q=Q_{H} \times Q_{G}$,

$-\delta((q, s), \sigma)=\delta_{H}(q, \sigma) \times \delta_{F}(q, \sigma)$,

$-F=\pi_{1}^{-1}\left(F_{H}\right) /\left(\pi_{2}^{-1}\left(F_{G}\right) \cap P^{*}\right)$.

Here, $P=\left\{(q, s) \mid q \in \delta_{H}^{*}(t), s \in \delta_{G}^{*}(t), t\right.$ a tree $\}$, the set of reachable states of the tree automaton $(Q, \delta, Q)$, which can be computed by a standard reachability algorithm.

We will now show that $L(R)=L / K$. Suppose $h_{1} \in L(R)$. Then $\delta^{*}\left(h_{1}\right) \cap F \neq \emptyset$ and we can take $\left(q_{1}, s_{1}\right) \cdots\left(q_{k}, s_{k}\right) \in \delta^{*}\left(h_{1}\right) \cap F$. By definition of $F$ there exists a word $\left(q_{k+1}, s_{k+1}\right) \cdots\left(q_{n}, s_{n}\right) \in \pi_{2}^{-1}\left(F_{G}\right) \cap P^{*}$ such that

$$
\left(q_{1}, s_{1}\right) \cdots\left(q_{k}, s_{k}\right)\left(q_{k+1}, s_{k+1}\right) \cdots\left(q_{n}, s_{n}\right) \in \pi_{1}^{-1}\left(F_{H}\right) .
$$

Hence, $q_{1} \cdots q_{n} \in F_{H}, s_{k+1} \cdots s_{n} \in F_{G}$, and $\left(q_{k+1}, s_{k+1}\right) \cdots\left(q_{n}, s_{n}\right) \in P^{*}$. By definition of $P$, there hence exists a hedge $h_{2}$ such that $q_{k+1} \cdots q_{n} \in \delta_{H}^{*}\left(h_{2}\right)$ and $s_{k+1} \cdots s_{n} \in \delta_{G}^{*}\left(h_{2}\right)$. Then $h_{2} \in L(G)=K$. Moreover, $h_{1} h_{2} \in L(H)=L$. Hence, $h_{1} \in L / K$, and thus $L / K \subseteq L(R)$. Conversely, let $h_{1}=\sigma_{1}\left[h_{1}^{\prime}\right] \cdots \sigma_{k}\left[h_{k}^{\prime}\right]$ be a hedge for which there exists some $h_{2}=\sigma_{k+1}\left[h_{k+1}^{\prime}\right] \cdots \sigma_{n}\left[h_{n}^{\prime}\right] \in K$ such that $h_{1} h_{2} \in L$. Then $\delta_{H}^{*}\left(h_{1} h_{2}\right) \cap F_{H} \neq \emptyset$ and $\delta_{G}^{*}\left(h_{2}\right) \cap F_{G} \neq \emptyset$. Hence we can choose $q_{1}, \cdots q_{n} \in$ $\delta_{H}^{*}\left(h_{1} h_{2}\right) \cap F_{H}$ and $s_{k+1} \cdots s_{n} \in \delta_{G}^{*}\left(h_{2}\right) \cap F_{G}$. Since $G$ is total there is at least one string $s_{1} \cdots s_{k} \in \delta_{G}^{*}\left(h_{1}\right)$. Hence, $s_{1} \cdots s_{n} \in \delta_{G}^{*}\left(h_{1} h_{2}\right)$ and $\left(q_{1}, s_{1}\right) \cdots\left(q_{n}, s_{n}\right) \in$ $\delta^{*}\left(h_{1} h_{2}\right)$. Since $\left(q_{k+1}, s_{k+1}\right) \cdots\left(q_{n}, s_{n}\right) \in \pi_{2}^{-1}\left(F_{G}\right)$ and since $\left(q_{1}, s_{1}\right) \cdots\left(q_{n}, s_{n}\right) \in$ $\pi_{1}^{-1}\left(F_{H}\right)$ we have $\left(q_{1}, s_{1}\right) \cdots\left(q_{k}, s_{k}\right) \in \pi_{1}^{-1}\left(F_{H}\right) /\left(\pi_{2}^{-1}\left(F_{G}\right) \cap P^{*}\right)$. Hence, $h_{1}$ is accepted by $R$, and thus $L / K \subseteq L(R)$.

An automaton for $K \backslash L$ can be constructed in a similar way. 